NBER WORKING PAPER SERIES

ASSET PRICING AND OPTIMAL PORTFOLIO

CHOICE IN THE PRESENCE OF ILLIQUID

DURABLE CONSUMPTION GOODS

Sanford J. Grossman

Guy Laroque

Working Paper No. 2369

NATIONAL BUREAU OF ECONOMIC RESEARCH

1050 Massachusetts Avenue

Cambridge, MA 02138

August 1987

We benefited from discussions with Yves Balasko. The research reported here is part of the NBER's research programs in Economic Fluctuations and Financial Markets and Monetary Economics. Any opinions expressed are those of the authors and not those of the National Bureau of Economic Research. 


\section{Asset Pricing and Optimal Portfolio Choice in the Presence of Illiquid Durable Consumption Goods}

\section{ABSTRACT}

We analyze a model of optimal consumption and portfolio selection in which consumption services are generated by holding a durable good. The durable good is illiquid in that a transaction cost must be paid when the good is sold. It is shown that optimal consumption is not a smooth function of wealth; it is optinal for the consumer to wait until a large change in wealth occurs before adjusting his consumption. As a consequence, the consumption based capital asset pricing model fails to hold. Nevertheless, it is shown that the standard, one factor, market portfolio based capital asset pricing model does hold in this environment.

It is shown that the optimal durable level is characterized by three numbers (not random variables), say $x, y$, and $z$ (where $x<y<z$ ). The consumer views the ratio of consumption to wealth (c/W) as his state variable. If this ratio is between $x$ and $z$, then he does not sell the durable. If $c / W$ is less than $x$ or greater than $z$, then he sells his durable and buys a new durable of size $S$ so that $S / W=y$. Thus $y$ is his "target" level of $\mathrm{c} / \mathrm{W}$. If the stock market moves up enough so that $\mathrm{c} / \mathrm{W}$ falls below $x$, then he sells his small durable to buy a larger durable. However, there will be many changes in the value of his wealth for which $c / W$ stays between $x$ and $z$, and thus consumption does not change.

Numerical simulations show that small transactions costs can make consumption changes occur very infrequently. Further, the effect of transactions costs on the demand for risky assets is substantial.

Sanford Grossman Department of Economics

Princeton University

Princeton, NJ 08544
Guy Laroque

INSEE

Paris, FRANCE 


\section{INTRODUCTION}

We analyze a model of optimal consumption and portfolio selection in which consumption services are generated by holding a durable good. The durable good is illiquid in that a transaction cost must be paid when the good is sold. It is shown that optimal consumption is not a smooth function of wealth; it is optimal for the consumer to wait until a large change in wealth occurs before adjusting his consumption. As a consequence, the consumption based capital asset pricing model (CCAPM) falls to hold. Nevertheless, it is shown that the standard, one factor, market portfolio based capital asset pricing model (CAPM) does hold in this environment.

In the standard model without transactions costs, and with additively separable utflity, a consumer, at an optimum, will be indifferent between investing a dollar and consuming goods worth a dollar. This implies that the derivative of his indirect utflity of wealth, say $V^{\prime}(W)$ will equal his marginal utility of consumption $u^{\prime}(c)$; call this the envelope condition. The CAPM is based upon noting that given two assets with returns $r_{f}$, and $r_{j}$, the consumer must be indifferent about switching a dollar from one to the other, and this implies that EV' $(W)\left(r_{i}-r_{j}\right)=0$. Roughly speaking, the CCAPM is derived from this equation by using the envelope condition. However, if it is costly to change the consumption flow, then it will no longer be the case that the envelope condition holds, and this breaks the link between the CCAPM and the CAPM.

A great deal of empirical evidence now exists in which the CCAPM is not only statistically rejected but also in which it is shown that the CAPM provides a better explanation for the observed risk premia on common stocks. 1 There are two sorts of inadequacies that are brought out by these studies. 
First, per capita consumption does not covary very much with stock returns, :o a very high risk aversion is needed to explain the observed risk premia of stocks. Second, the envelope condition forces the same parameter to be used for both intertemporal substitution and risk aversion, while the data suggests that two parameters are needed. 2 Both of these difficulties are avoided by considering a model where consumption derives from illiquid durables.

The structure and results of this paper are as follows. Section 2 states the consumer's optimization problem. It is assumed that the level of consumption services can be changed only by selling the existing durable (e.g., car or house) and purchasing a new one. In selling the old durable, a transactions cost must be paid which is proportional to the value of the durable being sold (e.g., a commission to a real estate broker). This acts like a fixed cost in an optimal stopping problem. The consumer can invest in $n$ risky assets and a risk free asset. There is no transactions cost involved in the purchase and sale of these financlal assets. 3 , The value of the risky assets follow a Brownian motion, and this is the source of randomness in the mode1. It is assumed that the consumer has a constant relative risk aversion utflity function over durable services.

In the absence of a transactions cost, the consumer would choose his consumption to maintain it in a fixed proportion to his wealth; if the stock market rises, then he increases his consumption, while if it falls, he decreases his consumption. Needless to say, this is not an optimal policy in the presence of a (fixed) transactions costs. It is surely suboptimal for a person to sell his house on every day in which the stock market changes, if on each such sale he must pay a broker a $5 \%$ transactions fee. It is thus obvious that the covariance of a single consumer's instantaneous consumption changes and stock returns will be zero most of the time, and hence not be 
proper measure of asset riskiness.

Section 3 provides a characterization of the optimal policies. It is shown that the optimal durable level is characterized by three numbers (not random variables), say $x, y$, and $z$ (where $x<y<z$ ). The consumer views the ratio of consumption to wealth $(c / W)$ as his state varlable. If this ratio is between $x$ and $z$, then he does not sell the durable. If $c / W$ is less than $x$ or greater than $z$, then he sells his house and buys a new house of size $S$ so that $S / W=y$. Thus $y$ is his "target" level of $c / W$. If the stock market moves up enough so that $c / w$ falls below $x$, then he sells his small house to buy a larger house. However, there will be many changes in i..e value of his wealth for which $c / W$ stays between $x$ and $z$, and thus consumption does not change.

Section 3 also proves that the consumer will choose a portfolio of stocks which is mean-variance efficient. As a consequence, equilibrium in the stock market requires that all consumers hold the market portfolio. This, of course, implies that the standard capital asset pricing model gives the risk premia of financial assets, f.e., an asset's mean excess return is proportional to its covariance with the return on the market portfolfo.

Finally, Section 3 discusses the extent to which transaction costs cause the consumer to act in a more risk averse manner with regard to his holdings of risky assets. It is shown that just after purchasing a new house, the consumer holds a smaller percentage of his wealth in risky assets than he would in the absence of transaction costs. This is because a random event, which increases his wealth, is worth less (than it would in the absence of transactions cost), since a cost must be borne to get the consumption benefits of the wealth increase. On the other hand, a random loss on the risky asset is now worse because not only does consumption have to fall but a 
transaction cost also must be paid (causing consumption to fall by more than it would have otherwise).

Section 4 presents numerical simulations of the model. It is shown that sma 11 transactions costs can make consumption changes occur very infrequently. Further, the effect of transactions costs on the demand for risky assets is substantial.

Section 5 contains conclusions, and a discussion of the empirical results of Bar-I lan and Blinder (1986) which bear directly on the model. The Appendix contains proofs of a 11 the Theorems. 


\section{STATEMENT OF THE CONSUMER'S OPTIMIZATION PROBLEM}

We assume that consumption services can be obtained only from the possession of a durable physical asset $K$. This yields a continuous flow of services to its owner and depreciates at rate $\alpha$ over time, $\alpha \geqslant 0$.

We study a situation which departs in two ma in ways from the standard consumption model. First, the physical asset comes in bulk ("houses") of various sizes, and the services accruing to a consumer comes from the house he lives in (the good is indivis ible once bought, and there is no rental market). Therefore, to change his consumption level beyond what is caused by depreciation, the consumer must sell his current house for a new one. Second, the market for houses may operate imperfectly, with transaction costs due to, e.g., costs of matching buyers and sellers. We model this imperfection by postulating that the selling price of the physical asset is a fraction $(1-\lambda)$ of its value, $0 \leqslant \lambda<1$. The case $\lambda=0$ corresponds to a perfect market. A similar description of the market for consumer durables has been used by flemming [1969].

In addition to the durable good, the consumer can invest his wealth into a risk free asset and a portfolio of risky assets. We take the durable good as the numeraire. We assume that the instantaneous return on the risk free asset is constant and given by $r_{f}$. Let $\hat{b}_{i t}$ be the value of the ith risky asset (inclusive of accumulated dividends) at time $t$. We assume that $d \hat{b}_{i t}=\hat{b}_{i t}\left(\hat{\mu}_{i} d t+d w_{i t}\right)$ where $w_{t} \equiv\left(w_{1 t}, w_{2 t}, \ldots, w_{n t}\right)$ is an $n$ dimensional brownian motion without drift, and instantaneous positive definite covarfance matrix $\Sigma$. Let $\underline{\hat{b}}_{t}=\left(\hat{b}_{1 t}, \hat{b}_{2 t}, \ldots, \hat{b}_{n t}\right)$ and $\hat{\underline{u}}=$ $\left(\hat{\mu}_{1}, \hat{\mu}_{2}, \ldots, \hat{\mu}_{n}\right)$. We assume that there are no transaction costs involved in buying or selling these financial assets. 
If we let $B_{t}$, and $\underline{x}_{t}$, respectively denote the (dollar) amount of the risk free asset and the vector of risky assets chosen by the consumer at time $t$, then his total wealth $Q_{t}$ satisfies

$$
Q_{t} \equiv K_{t}+B_{t}+\underline{x}_{t} \cdot \underline{l} \text {. }
$$

where $\underline{\ell}$ is a vector of 1 's. Let $\tau$ represent a time when the consumer sells his house. In any interval of time dt in which the consumer does not sell his house, his wealth evolves as

$$
d Q_{t}=-a K_{t} d t+r_{f} B_{t} d t+\underline{x}_{t} \cdot\left(\hat{\mu} d t_{t}+d \underline{w}_{t}\right) .
$$

Note that we can define $d \underline{b}_{t}=\left(\hat{\underline{u}-l_{r}}\right) d t+d \underline{w}_{t}$ to be the vector of excess returns on the risky assets, $\underline{\underline{\mu}} \equiv \hat{\underline{\mu}}-\underline{\ell} r_{f}$ as the vector of mean excess returns, and eliminate $B_{t}$ from $(2.1)$ and $(2.2)$ to get

$$
d Q_{t}=-\alpha K_{t} d t+r_{f}\left(Q_{t}-K_{t}\right) d t+\underline{x}_{t} \cdot d \underline{b}_{t} \quad \text { for } \tau \notin(t, t+d t) .
$$

If the consumer sells $h$ is house at time $\tau$, then

$$
Q_{\tau}=Q_{\tau^{-}}-\lambda K_{\tau^{-}}
$$

where $Q_{\tau^{-}}$refers to the level of $Q$ just before the house sale. Note that there is no transactions cost in purchasing a house, and $\lambda \mathrm{K}_{\tau^{-}}$is the loss in selling a house of size $\mathrm{K}_{\tau^{-}}$.

We consider an infinitely lived consumer in the economy whose tastes are represented by the expected value of an intertemporally separable utility function $E \int_{0}^{\infty} e^{-\delta t} u\left(K_{t}\right) d t$ where $\delta>0$, is the discount rate, and $K \geqslant 0$, is the quantity of durable good held at date $t$. The consumption service flow is taken to be proportional to the stock $k_{t}$.

Given inftial conditions $\left(Q_{0_{-}}, K_{0_{-}}\right)$, the problem of the consumer is to find non-anticipatory controls $\left(K_{t}, \underline{x}_{t}, t \geqslant 0\right) \quad\left(1 . e\right.$. , where $\left(K_{t}, x_{t}\right)$ 
depend only on the past values of $\left.\underline{b}\left(t^{\prime}\right), t^{\prime} \leqslant t\right)$, and non-anticipatory stopping times $\tau$ (i.e., he chooses a rule which determines for each time $t$ whether he should sell his house as a function of all the information he has up to time $t$ ) which maximize expected utility subject to $(2.3),(2.4)$ and a no bankruptcy constraint:

(2.5) $Q_{t}-\lambda K_{t} \geqslant 0 \quad$ for all $t$.

(If the consumer meets the constraint with equality at some date $t$, he is forced to sell his house at that date, and is left with a zero consumption from then on). We also assume that the absolute value of the fraction of wealth invested in any asset is bounded.

Let $V(Q, K)$ be the supremum of the expected utility that the consumer can achieve, from the initial conditions $(Q, K)$. We assume that the utility function exhibits constant relative risk aversion, f.e.:

$$
u(K)=\frac{k^{a}}{a} \quad \text { for some } a<1, a \neq 0 \text {, }
$$

and this enables us to reduce the problem from two state variables to a single state variable. The case of log utilfty, (1.e., $a=0$ ) can be analyzed as a separate case along identical lines as in the analys is to follow. Let

$$
\begin{aligned}
& \mu \equiv \mu \cdot \Sigma^{-1} \mu / \ell \cdot \Sigma^{-1} \cdot \mu \\
& \sigma^{2} \equiv \mu / \ell \cdot \Sigma^{-1} \cdot \underline{\mu} .
\end{aligned}
$$

Note that $\sigma^{2}>0$, and we assume that $\mu$ and $r_{f}+\alpha$ are strictly positive. In the Appendix we prove:

Theorem 2.1: Assume $B=\delta-a_{f}-\frac{\mu^{2}}{2 \sigma^{2}} \frac{a}{1-a}>0$, and let 
$v=\frac{1}{a\left(\alpha+r_{f}\right)}\left[\frac{\left(r_{f}+\alpha\right)(1-a)}{B}\right]^{1-a}$. Then $V(Q, K)$ is we 11 defined and there exists

a number $v_{2}>0$ such that

$v Q^{a} \geqslant V(Q, K) \geqslant \frac{v_{2}(Q-\lambda K)^{a}}{a}$

Furthermore, $V(Q, K)$ is homogenous of degree a in $(Q, K)$ and does not increase when $\lambda$ increases.

In all the following, we shall assume $B>0$ (otherwise in the absence of transaction costs, when $\lambda=0$, the consumer could achieve an infinite expected utility). The quantity $v$ in the above theorem is the utfilty the consumer gets when his initial wealth is equal to 1 and $\lambda=0$. It is immediate that the consumer cannot gain from an increase in transaction costs, and therefore $\vee Q^{\mathrm{a}}$ provides an upper bound for $V(Q, K)$. On the other hand, the following strategy is always available to the consumer: seil the house now and invest a 11 the proceedings in a new home to be kept forever without any intervention on the financial market. This gives the lower bound on $V(Q, K)$.

Now consider a consumer at date 0 , with initial conditions $(Q, K)$. If he decides to change houses immediately, he gets $\operatorname{Sup}_{C} V(Q-\lambda K, C)$. Therefore, if $V(Q, K)>\operatorname{Sup}_{C} V(Q-\lambda K, C)$ he will not change, while if the equality holds, he will change. Consequently, $V(Q, K)$ satisfles the following Belliman equation:

$$
V(Q, K)=\sup _{c, \tau,\left(\underline{X}_{t}\right)} E\left[\int_{0}^{\tau} e^{-\delta t} \frac{K_{t}^{a}}{a} d t+e^{-\delta \tau} V\left(Q_{\tau}-\lambda K_{\tau}, c\right)\right]
$$

where $\tau$ is the first stopping time from date 0 .

Using the homogeneity of $V(Q, K)$ we make a change of variable which 
enables us to reduce the problem to one state varfable $y$. Let:

$$
\begin{aligned}
y & =\frac{Q}{K}-\lambda \quad \underline{x}=\left(\frac{1}{K}\right) \underline{x} \\
h(y) & =K^{-a} V(Q, K) \\
\bar{\delta} & =\delta+a \alpha \quad r=\alpha+r_{f} .
\end{aligned}
$$

Substituting $K^{a} h(y)$ for $V(Q, K)$ into the Bellman equation gives:

$$
K^{a} h(y)=\operatorname{Sup}_{c, \tau,\left(\underline{x}_{t}\right)} E\left[\int_{0}^{\tau} e^{-\delta t\left(K e^{-\alpha t}\right)^{a}} \frac{a}{a} d t+e^{-\delta \tau} c^{a} h\left(\frac{Q_{\tau^{-}-\lambda K_{\tau^{-}}}}{c}-\lambda\right)\right]
$$

Let

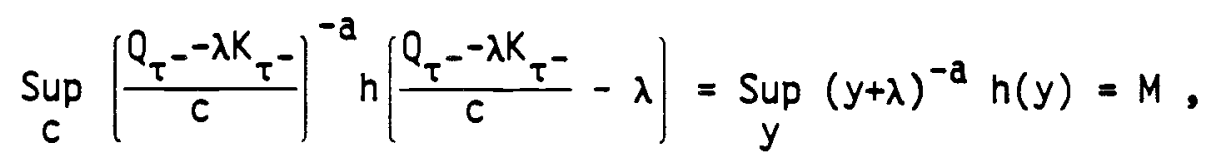

since $Q_{\tau^{+}}=Q_{\tau^{-}}-\lambda K_{\tau^{-}}$. Divide through by $K^{a}$, a positive number, and use the fact that $K_{\tau}=K e^{-\alpha \tau}$ to get

$$
h(y)=\operatorname{Sup}_{\tau,\left(\underline{x}_{t}\right)} E\left[\int_{0}^{\tau} \frac{e^{-\bar{\delta} t}}{a} d t+e^{-\bar{\delta} \tau} M y_{\tau^{-}}^{a}\right] .
$$

Since:

$$
\begin{aligned}
& d y=\frac{d Q}{K_{t}}-\frac{Q d K}{k_{t}^{2}} \text {, we obtain: } \\
& d y=\underline{x}_{t} \cdot d \underline{b}+r\left(y_{t}+\lambda-1\right) d t .
\end{aligned}
$$

The no bankruptcy constraint (2.5) becomes:

$$
\text { (2.11) } y_{t} \geqslant 0 \text { al1 } t \text {. }
$$

Finally, the definition of $M$ is: 


$$
M=\operatorname{Sup}_{y}(y+\lambda)^{-a} h(y)
$$

By Theorem 2.1, we have

$$
\operatorname{Sup}_{y}(y+\lambda)^{-a} v(y+\lambda)^{a} \geqslant M \geqslant \operatorname{Sup}_{y}(y+\lambda)^{-a} \frac{y^{a}}{a} v_{2} \text { and hence: }
$$

$$
v \geqslant M \geqslant \frac{v_{2}}{a} \text {. }
$$

Given $M$ satisfying (2.13), we first study the optimization problem in (2.9) subject to (2.10) and (2.11). This is a problem where stopping occurs only once and the consumer receives a payoff $\mathrm{My}^{\mathrm{a}}$ when he stops in state $y$. The following result is an application of Krylov [1980], p.39.

Theorem 2.2: Let $M$ be exogenously fixed satisfying (2.13), and let $h(y ; M)$ denote the solution to $(2.9)-(2.11)$ for such a given $M$. Then suppressing the dependence of $h(y ; M)$ on $M$ for notational convenience, $h(y)$ is continuously differentiable (except possibly at $y=1-\lambda$ ) and:

(i) If $h(y)>y^{a} M$, then it is optimal to not stop (f.e., $\tau \neq 0$ ), $h(y)$ is twice continuously differentiable except possibly at $y=1-\lambda$, and (2.14a) $\operatorname{Sup}_{\underline{x}}\left[\frac{h^{\prime \prime}(y)}{2} \operatorname{Var} d y+h^{\prime}(y) E d y-\bar{\delta} h(y)+1 / a\right]=0$. (2.14b) where $\operatorname{Var} d y \equiv \underline{x}^{\prime} \sum \underline{x}$ and $E d y \equiv r(y+\lambda-1)+\underline{x} \cdot \underline{\mu}$.

(ii) If $h(y)=y^{a} M$, then stop (i.e., $\tau=0$ ) and

(2.15) $\operatorname{Sup}_{\underline{x}}\left[\frac{h^{\prime \prime}(y)}{2} \operatorname{Var} d y+h^{\prime}(y) E d y-\bar{\delta} h(y)+1 / a\right] \leqslant 0$, except possibly at the boundary points of the set $\left\{y \mid h(y)>y^{a} M\right\}$; (2.16) $\quad h^{\prime}(y)=a y^{a-1} M \quad$ if $h(y)=y^{a} M$. 
The portfolio $\underline{x}(y)$ is optimal at state $y$ if it attains the supremum in (2.14).

This theorem can be understood as follows. First, from (2.9) it is clear that $h(y) \geq \mathrm{My}^{\mathrm{a}}$ since it is a iways feasible to set $\tau=0$. If $h(y)>M y^{a}$ then this means that $\tau \neq 0$, so (roughly speaking) by continuity, there is a time $t$ small enough so that we can ignore events where stopping occurs during $(0, t)$ and thus:

$$
h(y)=\sup _{\underline{x}} E\left[\int_{0}^{t} \frac{e^{-\overline{\delta s}}}{a} d s+e^{-\bar{\delta} t} h\left(y_{t}\right)\right] \text {, }
$$

and therefore, bringing the left-hand side to the right-hand side, and dividing by $t$ :

$$
0=\operatorname{limit}_{t \rightarrow 0} \sup _{\underline{x}} E\left[\frac{1}{t} \int_{0}^{t} \frac{e^{-\bar{\delta} s}}{a} d s+\frac{1}{t}\left(e^{-\bar{\delta} t} h\left(y_{t}\right)-h(y)\right)\right] .
$$

However, if we let $z_{t} \equiv e^{-\bar{\delta} t} n\left(y_{t}\right)$, then

$$
\lim _{t \rightarrow 0} \frac{1}{t} E\left(e^{-\bar{\delta} t} h\left(y_{t}\right)-h(y)\right) \equiv \lim _{t \rightarrow 0} \frac{E Z_{t}-z_{0}}{t} \equiv E d Z
$$

where EdZ at $t=0$ is found by Ito's Lemma to be

$$
h^{\prime}(y) E d y+\frac{1}{2} h^{\prime \prime}(y) \operatorname{Var} d y-\bar{\delta} h(y) \text {. }
$$

This, combined with $\lim _{t \rightarrow 0} \frac{1}{t} \int_{0}^{t} \frac{e^{-\overline{\delta s}}}{a} d s=1 / a \quad$ gives (2.14).

The theorem states that there will be regions of values for $y$ where $h(y)>y^{a} M$ and no stopping is optimal, and other regions where stopping is optimal and $h(y)=y^{a} M$. Condition (2.16) is the "smooth pasting" condition 
which requires that $h(y)$ be differentiable at the boundartes of regions where $h(y)>M y^{a}$.

In the next Section we show that there is only one connected region where $h(y)>y^{a} M$, and we characterize the optimal portfolio rules. 


\section{Optima 1 Portfolio and Consumption Rules}

We begin by showing that the consumer chooses a mean-variance effictent portfolio. This result does not require that the utility function exhibit constant relative risk aversion. 4

Theorem 3.1 In state $y$, the consumer chooses a portfolto $\underline{x}(y)$ which maximizes $\underline{x} \mu+\frac{h^{\prime \prime}(y)}{2 h^{\prime}(y)} \underline{x} \cdot[\cdot \underline{x}$, and consequent $1 y$

$$
\underline{x}(y)=\frac{-h^{\prime}(y)}{h^{\prime \prime}(y)} \Sigma^{-1} \cdot \underline{\mu} .
$$

Proof: This follows immediately from Theorem 2.2, and the fact, proved in the Appendix, that $\frac{h^{\prime}(y)}{h^{\prime \prime}(y)}<0$, by substituting (2.14b) into (2.14a) and maximizing with respect to $\underline{x}$.

Since al1 consumers hold risky assets in the same proportion (even if they have different utflity functions and values of $y$ ), and since financial market clearing implies that the sum of their holdings must equal the value of the market portfolio, we obtain the standard (market portfolio based single factor) capital asset pricing mode1:

Theorem 3.2 Let $r_{m}$ be the instantaneous return on the market portfolito (i.e., a portfolto where the th financial asset has weight $\hat{b}_{i} / \sum_{j=1}^{p} \hat{b}_{j}$ ), then a necessary condition for market clearing is

$$
\mu_{1}=\frac{\operatorname{Cov}\left(\frac{d \hat{b}_{i}}{\hat{b}_{i}}, r_{m}\right)}{\operatorname{Var}\left(r_{m}\right)}\left(E r_{m}-r_{f}\right) \quad i=1,2, \ldots, n .
$$

Proof: Recal1 that $\underline{x}(y)=\frac{1}{K} \underline{x}(y)$, so that (3.1) implies that $\underline{X}(y)=s(y, K) \Sigma^{-1} \mu$, where $s(y, K)$ is a positive scalar which depends on the consumer's tastes, $y$ and $K$. This can be summed over consumers to yield $\underline{\hat{b}}_{t}=s_{2 t} \Sigma^{-1} \underline{\mu}$, where $s_{2 t}$ is a positive scalar and $\hat{\underline{b}}_{t}$ is the vector of 
asset values. This impiles that

$$
\underline{\mu}=\frac{1}{s_{2 t}} \Sigma \cdot \underline{\hat{b}}_{t} \quad \text { and therefore } \quad s_{2 t}=\underline{\hat{b}}_{t} \cdot \sum \cdot \hat{\underline{b}}_{t} / \underline{\hat{b}}_{t} \cdot \underline{\mu}
$$

and these two equations combine to give the result to be proved, since $\sum$ is a matrix with (i.j) element equal to $\operatorname{Cov}\left(d \hat{b}_{j} / \hat{b}_{i}, d \hat{b}_{j} / \hat{b}_{j}\right)$.

Theorem 3.2 allows us to change the control in (2.9), (2.10), (2.14) and (2.15) from the vector $\underline{x}$ to a scalar $x$ which denotes the amount invested in the market portfolio. Note that (2.6) gives the mean rate of return $\mu$ and variance $\sigma^{2}$ of the market portfolio. Therefore, (3.1) becomes

$$
x(y)=\frac{-h^{\prime}(y)}{h^{\prime \prime}(y)} \frac{\mu}{\sigma^{2}}
$$

and $\operatorname{Var} d y=x^{2} \sigma^{2}$ and $E d y=r(y+\lambda-1)+x \mu$ in (2.14b).

In order to understand why the consumption based asset pricing mode 1 fails in our context it is necessary to further characterize the optimal consumption policy. The next theorem states that the optimal consumption policy is characterized by three numbers $y_{1}, y^{*}$, and $y_{2}$ with the property that $y_{1} \leq y^{*} \leq y_{2}$ and if the state variable $y$ is between $y_{1}$ and $y_{2}$, then the house is not sold; if $y$ is not between $y_{1}$ and $y_{2}$, then the house is sold and a new house is purchased to bring the level of the state variable from $y$ to $y^{\star}$.

Theorem 3.3: There exists three numbers $y_{1} \leq y^{\star} \leq y_{2}$ such that $h(y)$ in Theorem 2.2 satisfies

$$
h(y)>M y^{a} \quad \text { if and only if } y \in\left(y_{1}, y_{2}\right) \text {, }
$$

and 


$$
M=\left(y^{*}+\lambda\right)^{-a} h\left(y^{*}\right)=\sup _{y}(y+\lambda)^{-a} h(y) .
$$

To understand this recall that $y=\frac{Q}{K}-\lambda$. If the financlal assets $r$ ise in value, then $Q$ will rise and eventually the consumer will feel sufficlently wealthy that he wants a larger house. When $Q$ rises to the point where $y=y_{2}$, the consumer purchases a house $K^{\star}(Q)$ with the property that his new ratio of wealth to housing $Q / K^{\star}(Q)$ satisfies

$$
Q / K^{*}(Q)-\lambda=y^{*}
$$

A similar effect appears on the downside. If wealth falls sufficiently, then the old house will be sold and a new house will be purchased which satisfies (3.4). Note that the size of the new house depends only on the total wealth Q. Note that if $Q_{\tau-}$ was the level of wealth just before switching houses, then the $Q$ in (3.4) is $Q_{\tau_{-}}-\lambda K_{\tau_{-}}$.

We are now in a position to give a complete mathematical description of the solution. Using (3.2) and (2.14), we seek a function $h(\cdot)$ and numbers $M, y_{1}, y^{*}, y_{2}$ such that

$$
-\frac{1}{2}\left(\frac{\mu}{\sigma}\right)^{2} \frac{\left(h^{\prime}(y)\right)^{2}}{h^{\prime \prime}(y)}+r(y+\lambda-1) h^{\prime}(y)-\bar{\delta}(y)+\frac{1}{a}=0
$$

$$
\text { for } y \in\left[y_{1}, y_{2}\right] \text {; }
$$

$$
h(y) \geq y^{a} M \quad \text { for all } y \text {; }
$$

and from (2.16)

$$
\begin{aligned}
& h\left(y_{j}\right)=y_{i}^{a} M \quad \text { for } \quad i=1,2 ; \\
& h^{\prime}\left(y_{i}\right)=a y_{i}^{a-1} M \quad \text { for } i=1,2 ;
\end{aligned}
$$

and from (3.4) 


$$
M=\left(y^{\star}+\lambda\right)^{-a} h\left(y^{\star}\right)=\sup _{y}(y+\lambda)^{-a} h(y) .
$$

To understand this system fix $M$ and ignore (3.9). Equations (3.7) and (3.8) represent 2 free boundary conditions for the second order differential equation (3.5). Such a system can be understood by picking a guess for $y_{1}$, then using (3.7) and (3.8) to get $h\left(y_{1}\right)$ and $h^{\prime}\left(y_{1}\right)$. This provides an initial condition to (3.5). Then (3.5) can be continued while $h(y)>y^{a} M$ until a point $y_{2}$ is found where $h\left(y_{2}\right)=y_{2}^{a}$ M. If it is also the case that $h^{\prime}\left(y_{2}\right)=a y_{2}^{a-1} M$, then we are done, otherwise choose a new value of $y_{1}$ and repeat. If a solution can be found, then for the fixed $M_{\text {, }}$; we will have a solution to (3.5) - (3.8): $h(y ; M)$. This function is then substituted into (3.9) to yield the problem: find an $M^{*}$ such that

$$
M^{*}=\sup _{y}(y+\lambda)^{-a} h\left(y ; M^{*}\right)
$$

The value for $y^{\star}$ is the $y$ which attains the supremum in (3.10).

We do not have an analytic solution to (3.5)-(3.9); however, the appendix shows that there exists a solution and the next Section presents numerical simulations of the solution. In addition, we prove that as transaction costs increase, the interval over which no house sale occurs $\left(y_{1}, y_{2}\right)$ grows:

Theorem 3.4: If $y_{1}, y_{2}, M$ solve (3.5) - (3.9), then $y_{1}$ is a strictly decreasing function of $\lambda$, and $y_{2}$ is a strictly increasing function of $\lambda$. If $M>1 /(a \bar{\delta})$, then $M$ is a strictly decreasing function of $\lambda$.

Note that $M=1 /(\mathrm{a} \bar{\delta})$ when 1 it is optimal to never sell a house, starting from $y=y^{\star}$, i.e. $y^{\star}=1-\lambda, x\left(y^{\star}\right)=0$ and neither $y_{1}$ nor $y_{2}$ are ever reached starting from $y=y^{\star}$. 
Though we cannot explicitly solve for $h(\cdot)$, substituting (3.2) into (3.5) and differentiating once can be used to show that $x(y)$ satisfies the following differential equation

$$
\begin{gathered}
\frac{\mu}{2} x^{\prime}(y) x(y)+\left(r-\bar{\delta}-\frac{\mu^{2}}{2 \sigma^{2}}\right) x(y)-\frac{\mu r}{\sigma^{2}}(y+\lambda-1)=0 \\
\text { for } y \in\left(y_{1}, y_{2}\right) .
\end{gathered}
$$

Theorem 3.5 Let

$$
\gamma=\left(\bar{\delta}+\frac{\mu^{2}}{2 \sigma^{2}}-r\right) / \sqrt{\left(\bar{\delta}+\frac{\mu^{2}}{2 \sigma^{2}}-r\right)^{2}+2 r \frac{\mu^{2}}{\sigma^{2}}}
$$

and let $\theta_{1}<0, \theta_{2}>0$ be the two roots of the second degree equation

$$
\theta^{2}+2\left[\frac{r-\bar{\delta}}{\mu}-\frac{\mu}{2 \sigma^{2}}\right] \theta-2 \frac{r}{\sigma^{2}}=0 .
$$

Then the optimal $x$ at $y, x(y)$ satisfies:

(3.14a) $\quad x(y) \geq \operatorname{Max}\left(\theta_{1}(y+\lambda-1), \theta_{2}(y+\lambda-1)\right)$

$$
\left(x(y)-\theta_{1}(y+\lambda-1)\right)^{1-\gamma}\left(x(y)-\theta_{2}(y+\lambda-1)\right)^{1+\gamma}=c
$$

for some real number $c \geq 0$. Note that $\gamma \in[0,1]$.

To understand (3.14) make a change of variable to $z=y+\lambda-1$ and $\bar{x}(z)=x(z+1-\lambda)$, so $(3.14)$ becomes

$$
\left(\bar{x}(z)-\theta_{1} z\right)^{1-\gamma}\left(\bar{x}(z)-\theta_{2} z\right)^{1+\gamma}=c \text { for } z \in\left(z_{1}, z_{2}\right)
$$

where $z_{1}=y_{1}+\lambda-1$ and $z_{2}=y_{2}+\lambda-1$.

Figure 1 plots the lines $\bar{x}=\theta_{1} z /(z+1)$ and $\bar{x}=\theta_{2} z /(z+1)$. Also plotted, for 3 values of $\lambda$, is the curve $\bar{x}(z) /(z+1)$ which lies above these two 7 ines. As $c$ goes to zero, the curve $\bar{x}(z)$ collapses onto the curve formed by the two lines $\bar{x}=\theta_{1} z$ and $\bar{x}=\theta_{2} z$. Note that $\bar{x}(z) /(z+1)$ represents the fraction of wealth $x / Q$ invested in the risky 
asset. Note that from (2.1) and (2.8), $z=(X+B) / K$, and thus unlike $y, z$ is a description of the state independent of $\lambda$. Hence, the reaction of the $x(z)$ to a change in $\lambda$ tells us how the holdings of risky assets change in a particular state when $\lambda$ changes.

Note that the left-hand side of (3.15) is independent of $\lambda$ (i.e., $\theta_{1}, \theta_{2}$ and $\gamma$ do not depend on $\lambda$ ). Therefore, if we compare the solutions to two identical optimization problems, except that $\lambda=\lambda_{a}$ in one and $\lambda=\lambda_{b}$ in the other, then only $c$ can be different across the two problems. If we denote the optimal value of $\bar{x}(z)$ by $x(z ; \lambda)$ to indicate its dependence on $\lambda$, then the previous remarks imply that $\bar{x}\left(z ; \lambda_{a}\right)$ is either everywhere above or everywhere below $\bar{x}\left(2 ; \lambda_{b}\right)$ for the range of $z^{\prime}$ 's where (3.15) holds. We do not know that $c$ varies monotonically with $\lambda$. This is illustrated in Figure 1 where $\lambda_{a}>\lambda_{b}$ and $\bar{x}\left(z ; \lambda_{a}\right)$ lies everywhere below $\bar{x}\left(z, \lambda_{b}\right)$. Note, however, that there are values for $\bar{x}\left(\cdot ; \lambda_{a}\right)$ which are larger than $\bar{x}\left(\cdot ; \lambda_{b}\right)$. For example $x\left(z_{1 a} ; \lambda_{a}\right)>$ $x\left(z ; \lambda_{b}\right)$ for all $z \in\left[z_{1 b}, z_{2 b}\right]$. Note that if $\lambda=\lambda_{b}$ then $z=z_{1 a}$ is not an attainable point in the state space since the consumer sells his house at any state below $z_{1 b}$.

The above remarks indicate that the effect of $\lambda$ on the holdings of risky assets is complex, and dependent on the particular state that the consumer is in. Roughly speaking, the existence of transactions costs makes the consumer more risk averse in the middle of his state space (1.e., near $y=y^{*}$ ) and less risk averse at the boundaries of his state space ( $y=y_{1}$ or $\left.y=y_{2}\right)$. This can be understood by noting that the consumer's direct utfifty function $M^{a}$ is replaced by his indirect utflity function $h(y)$ for purposes of asset choice. Figure 2 fllustrates the consequences of this by plotting $h(y)-M y^{a}$. Note that the smooth pasting conditions assure that 
$h(y)$ is tangent to $M y^{a}$ at $y=y_{1}$ and $y=y_{2}$. As the Figure shows, this implies that $h(y)$ is less concave than $M y^{a}$ at these points. (The curve may not appear tangent at $y_{1}$, but it is tangent; the second derivative of $h(y)-M y^{a}$ is quite large just to the right of $y_{1}$.) Clearly however, $h(y)$ must become more concave than $M y^{a}$ somewhere between $y_{1}$ and $\mathrm{y}_{2} \cdot$

To explore this further, note that

(3.16) $\quad \frac{x(y)}{y}=\frac{x}{Q-\lambda K}$.

gives the proportion of marketable wealth invested in the risky asset. If $\lambda=0$, then our model is equivalent to Merton's model where the proportion of wealth invested in the risky asset is

(3.17) $\quad \frac{X}{Q}=\frac{\mu}{(1-a) \sigma^{2}}$

We are able to prove the following:

Theorem 3.6: $\quad \frac{x\left(y_{1}\right)}{y_{1}} \geq \frac{\mu}{(1-a) \sigma^{2}} \quad 1=1,2$

$$
\frac{x\left(y^{\star}\right)}{y^{\star}} \leqslant \frac{\mu}{(1-a) \sigma^{2}} \text {, }
$$

Therefore, the consumer behaves in a more risk averse manner just after purchasing a new house, and in a less risk averse manner just before purchasing a new house. 


\section{NUMERICAL SIMULATIONS}

The boundary value problem (3.5)-(3.9) can be solved on a computer using roughly the technique described in Section 3 just after (3.9). We make the following assumptions about parameters: The mean and standard deviation of the excess return on the market portfolto are $5.9 \%$ and $22 \%$ respectively from Ibbotson and Sinquefeld (1982).

Since the durable good is the numeraire, the nominal inflation on durable goods should be subtracted from the nominal rate of interest to get the appropriate real rate, $r_{f}$. Ibbotson and Sinquefeld report an average short run rate of $4.3 \%$ between 1953 and 1979 . In the same period the nomina 7 inflation in housing prices was $4.2 \%$ per year, and $2.5 \%$ per year for automobiles. 5 We thus, somewhat arbitrarily, set the risk free rate $r_{f}$ to be $1 \%$.

Table 1 presents some numerical results for the case of no depreciation $(\alpha=0), \delta=r_{f}$, and for various values of $\lambda$, and of $A \equiv 1-a$ which is the coefficient of relative risk aversion. In order to define state varlables independent of $\lambda$, we let

(4.1) $\bar{y} \equiv y+\lambda=Q / K$.

Column 3 gives the left boundary $\bar{y}_{1}$, and the right boundary $\bar{y}_{2}$ of the no stopping region. It also gives the point $\bar{y}^{\star}$ to which $Q / K$ is brought after $\bar{y}$ hits a boundary point.

Column 4 presents the expected length of time between house purchases. Following Karlin and Taylor [1981,p.192] let $T_{y}$ be the length of time it takes to hit a boundary starting from $y$, and (4.2) $\quad V_{a}(y)=E T_{y}$, 
Then $V_{a}()$ satisfies the following differential equation

$$
-1=V_{a}^{\prime}(y) E d y+\frac{1}{2} V_{a}^{\prime \prime}(y) \operatorname{Var} d y,
$$

with boundary conditions $v_{a}\left(y_{1}\right)=v_{a}\left(y_{2}\right)=0$.

This is solved numerically, and $v_{a}\left(y^{\star}\right)$ appears in the table.

The fifth column presents the result of numerical calculations on the average holding of risky assets. The fraction of wealth invested in the risky asset is a function of the state $y$. This state is a renewal process which goes from $y^{\star}$ to a boundary and then returns. Karlin and Taylor [1981, pp.192, 261] shows that the expected value of any function $f(\cdot)$ of the state, can be found by solving the differential equation (4.4) $\quad-f(y)=V_{b}^{\prime}(y) E d y+\frac{1}{2} V_{b}^{\prime \prime}(y)$ Var $y$ for $V_{b}(\cdot)$ with boundary conditions $V_{b}\left(y_{1}\right)=V_{b}\left(y_{2}\right)=0$. The average value of $f(\cdot)$ over renewal cycles is $v_{b}\left(y^{\star}\right) / v_{a}\left(y^{\star}\right)$. In column 5 , the function presented is (4.5) $\quad f(y)=\frac{X}{Q}(y) \equiv \frac{X}{K} \cdot \frac{K}{Q} \equiv \frac{X(y)}{y+\lambda}$. which represents the fraction of total wealth invested in risky assets.

The sixth column uses wealth net of the transactions cost of selling the durable to compute the average fraction of wealth held in the form of the risky asset. This is computed by setting $f(y)=x(y) / y$ in the previous calculation.

The seventh column gives the value of $X / Q$ in the absence of transactions cost (i.e. in Merton's mode1), as it appears in (3.17).

The eighth column gives the probability that the consumer will reach the lower boundary before reaching the upper boundary, given that he starts from $y=y^{\star}$. It thus gives the fraction of occasions that a change in the value of the stock market causes people to buy smaller houses, rather than larger houses. 
The ninth (and final) column gives the rate of $\mathrm{drift}$ in wealth evaluated at $y=y^{\star}$. It is computed as $\frac{1}{y} E d y=\frac{\mu x(y)+(y+\lambda-1) r}{y}$. Discussion of Table 1

As is expected, a rise in transactions cost increases the average time between durable sales. The average time between durable sales is quite large even for very small transactions costs. Note that in this infinite horizon model, if risky assets were not held (1.e., if $\mu=0$ ) and $r=\bar{\delta}$, then the consumer starting from $y^{\star}$ would never switch. He would invest all of his wealth in housing and consume the service flow. It is the uncertainty about stock returns and the upward drift in wealth when $\mu>0$ that causes the boundaries to be hit.

In particular, with $\delta=.01, r=.01$ and $\mu=.059$ and the range of risk aversions being considered, the returns from saving in the form of financial assets are so large that the consumer chooses a lifetime consumption profile which (on average) drifts upward. To accomplish this he chooses a relatively small durable and relatively large financial investments with the property that his wealth drifts upward in the period between durable purchases. 6 ,

Hence, the major reason for durable sales is the upward drift in wealth. As the final column of the Table makes clear, the parameters chosen imply that wealth is expected to $r$ ise at a rate of, say, $6.22 \%$ for $A=1.1$ and $\lambda=.05$ just after a new durable purchase has been made.

It should be emphasized that we are considering durable good sales caused only by changes in wealth; not caused by death, switching of jobs or spouses, or changes in family size. The point to realize is that changes in stock market wealth will be associated with consumption changes for an individual only when measured over decades; there is essentially no covariance between 
consumption changes and stock returns on a monthly or annual basis for realistic measures of transactions cost.

The Table shows that the average fraction of wealth invested in risky assets, $X / Q$, falls as transactions costs rise. For $A=2.0$, the average holdings of risky assets falls from .584 at $\lambda=.005$ to .460 at $\lambda=.25$; in each case it is substantfally lower than the no transactions cost case where $\lambda=0$.

The next column on the Table, labeled $\left(E_{T}\right)^{-1} E X /(Q-\lambda K)$, also computes the average fraction of "wealth" invested in the risky asset, but "wealth" refers to the amount of money that would be realized if all assets and durables were liquidated. This measure of wealth depends on the level of transactions cost. The table shows that the average fraction invested in risky assets is not a monotone function of $\lambda$. When $\lambda$ is large, "wea 1 th" falls and this makes it appear as if a large fraction of "wealth" is invested in the risky asset.

It is interesting to note that, for a given $\lambda$, as the consumer becomes less risk averse (1.e., A falls) the average holdings of risky assets gets closer to the $\lambda=0$ risky asset level. We understand this to be caused by the fact that when $A$ falls the consumer holds more risky assets, and hence spends more time near the boundaries of his "no stopping" region. (Note the ET falls as A falls.) The transactions cost causes $\mathrm{h} 1 \mathrm{~m}$ to be less risk averse near the boundaries than he would be if $\lambda=0$, as noted in Section 3.

Discussion of Tables 2,3 , and 4

These tables consider the same parameters as Table 1, except that $\delta$ is raised to $2 \%, 4 \%$, and $6 \%$ : in Tables 2,3 and 4 respectively. It may be thought that raising the discount rate lowers the average time between 
durable sales. The reasoning may be that when the discount rate is high then the consumer should take advantage of a rise in wealth to increase his consumption sooner rather than later. A comparison of Table 2 and Table 1 shows that this is not the case. Instead, the dominant effect appears to be that a rise in the discount rate to $2 \%$ lowers investment in financial assets (as can be seen by comparing $\left(\bar{y}_{1}, \bar{y}^{\star}, \bar{y}_{2}\right.$ ) across the two tables), and this lowers the rate of upward drift in wealth.

Table 4 , in which $\delta=6 \%$, shows that the investment in financial assets falls to the point where the expected drift in wealth is almost zero just after a new durable is purchased. For such parameter values, the purchase of a durable, in the steady state, is due to variability in wealth rather than the drift in wealth. The expected time between purchases is very large, far larger than for the $\delta=1 \%$ of Table 1 . Thus, for a consumer in a steady state (i.e., where wealth would not be expected to drift after a purchase), the variability of the stock market would not be correlated with an infinitely long lived consumer's durable purchase except at frequences of many decades. Discussion of Table 5

The previous discussion concerned situations where the durable does not depreciate. If the durable depreciates rapidly, then there will be very frequent purchases. The first column of Table 5 gives various annual depreciation rates of the durable good from $\alpha=0$ to $\alpha=.10$. Note that the average fraction of wealth invested in the risky asset becomes very close to its $\lambda=0$ value when $\alpha$ is large. Further, it should be clear that consumption will be a more responsive function of wealth when rapld depreciation causes new purchases to occur very frequently. 
$6 / 01 / 87$

\section{Table 1}

Some Numerical Simulations

\begin{tabular}{|c|c|c|c|c|c|c|c|c|c|}
\hline$\lambda$ & A & $\left(\bar{y}_{1}, \bar{y}^{*}, \bar{y}\right.$ & & $\begin{array}{c}\text { (Years) } \\
E_{T}\end{array}$ & $\frac{E(X / Q)}{E(\tau)}$ & $\frac{E\left[\frac{x}{Q-\lambda K}\right]}{E(\tau)}$ & $\begin{array}{c}\lambda=0, \\
x / 0\end{array}$ & $\begin{array}{l}\text { Prob. } \\
\text { (buy- } \\
\text { down) }\end{array}$ & $\begin{array}{l}\text { Rate of } \\
\text { Wealth } \\
\text { Drift } \\
\text { at } y^{\star}\end{array}$ \\
\hline .005 & 2.0 & $(.34,0.58$, & $0.89)$ & 11.47 & 0.584 & 0.587 & 0.610 & .226 & .0262 \\
\hline .25 & 2.0 & $(.40,0.92$, & 2.54) & 47.42 & 0.460 & 0.604 & 0.610 & .022 & .0293 \\
\hline .05 & 2.0 & $(.29,0.70$ & $1.43)$ & 28.94 & 0.530 & 0.570 & 0.610 & .093 & .0258 \\
\hline .05 & 1.75 & $(.27,0.71$ & $1.56)$ & 27.33 & 0.609 & 0.654 & 0.697 & .100 & .0313 \\
\hline .05 & 1.5 & $(.26,0.76$, & 1.81) & 25.64 & 0.717 & 0.768 & 0.813 & .107 & .0391 \\
\hline .05 & 1.1 & $(.26,1.08$ & $3.16)$ & 22.39 & 1.015 & 1.069 & 1.108 & .121 & .0622 \\
\hline 05 & 0.9 & $(.44,2.56$, & $8.71)$ & 20.46 & 1.303 & 1.335 & 1.354 & .129 & .0845 \\
\hline .005 & 0.9 & $(.76,2.03$ & $4.38)$ & 7.832 & 1.342 & 1.345 & 1.354 & .261 & .0840 \\
\hline .25 & 0.9 & $(.39,3.62$, & $18.30)$ & 35.08 & 1.220 & 1.342 & 1.354 & .042 & .0875 \\
\hline .08 & 2.0 & $(.30,0.74$, & 1.65) & 33.93 & 0.511 & 0.570 & 0.610 & .069 & .0262 \\
\hline .08 & 1.75 & $(.28,0.76$ & $1.82)$ & 32.17 & 0.587 & 0.654 & 0.697 & .074 & .0317 \\
\hline .08 & 1.5 & $(.26,0.81$, & 2.12) & 30.16 & 0.693 & 0.768 & 0.813 & .082 & .0397 \\
\hline .08 & 1.1 & $(.26,1.17$, & $3.78)$ & 26.49 & 0.989 & 1.068 & 1.108 & .095 & .0630 \\
\hline .08 & 0.9 & $(.40,2.78$, & 10.56) & 24.19 & 1.286 & 1.334 & 1.354 & .103 & .0849 \\
\hline 10 & 2.0 & $(.31,0.11$, & $1.71)$ & 36.48 & 0.501 & 0.572 & 0.610 & .058 & .0264 \\
\hline 10 & 1.75 & $(.29,0.79$, & 1.97) & 34.62 & 0.577 & 0.656 & 0.697 & .063 & .0321 \\
\hline 10 & 1.5 & $(.27,0.85$, & $2.31)$ & 32.49 & 0.680 & 0.770 & 0.813 & .070 & .0402 \\
\hline .10 & 1.1 & $(.27,1.22$, & 4.14) & 28.50 & 0.975 & 1.069 & 1.108 & .083 & .0635 \\
\hline .10 & 0.9 & $(.37,2.90$ & 11.66) & 26.34 & 1.276 & 1.334 & 1.354 & .088 & .0852 \\
\hline
\end{tabular}

Notes: $\alpha=0, r_{F}=.01, \delta=0.01, \mu=.059, \sigma=.22$

$100 \lambda$ is the percent transactions cost of selling the old durable;

$A \equiv 1-a \quad$ is the coefficient of relative risk aversion;

$\left(\bar{y}_{1}, \bar{y}^{\star}, \bar{y}_{2}\right) \quad$ are the three values of $Q / K$ which characterize optima 1 stopping;

$E_{\tau}$ is the expected length of time to reach either $\bar{y}_{1}$ or $\bar{y}_{2}$ starting from $\bar{y}^{\star}$; 
Table 1 (continued)

$$
\begin{array}{ll}
\left(E_{\tau}\right)^{-1} E X / Q & \begin{array}{l}
\text { is the average fraction of wealth invested in the risky } \\
\text { asset, using the steady state distribution under the } \\
\text { optima } 7 \text { policy; }
\end{array} \\
\left(E_{\tau}\right)^{-1} E[X /(Q-\lambda K)] & \begin{array}{l}
\text { is the same as }\left(E_{\tau}\right)^{-1} E X / Q \text { except that "wealth" refers } \\
\text { to the amount which is obtained net of transactions cost } \\
\text { when all assets and durables are sold. }
\end{array} \\
\begin{array}{ll}
\text { Prob (buy down) } & \begin{array}{l}
\text { is the value of } X / Q \text { in the absence of transactions cost. } \\
\text { is reached; }
\end{array}
\end{array}
\end{array}
$$

\begin{tabular}{|c|c|c|c|c|c|c|c|c|}
\hline$\lambda$ & A & $\left(\bar{y}_{1}, \bar{y}_{0}, \bar{y}_{2}\right)$ & $\begin{array}{c}\text { (Years) } \\
E_{\tau}\end{array}$ & $\frac{E(X / 0)}{E(\tau)}$ & $\frac{E\left[\frac{X}{0-\lambda K}\right]}{E(\tau)}$ & $\begin{array}{c}\lambda=0 \\
x / Q\end{array}$ & $\begin{array}{l}\text { Prob. } \\
\text { (buy- } \\
\text { down) }\end{array}$ & $\begin{array}{l}\text { Rate of } \\
\text { Wealth } \\
\text { Drift } \\
\text { at } y^{\star}\end{array}$ \\
\hline .005 & 2.0 & $(.274,0.465,0.711)$ & 11.86 & 0.576 & 0.583 & 0.610 & .274 & .0212 \\
\hline .25 & 2.0 & $(.375,0.796,2.162)$ & 56.90 & 0.403 & 0.565 & 0.610 & .050 & .0245 \\
\hline .05 & 2.0 & $(.247,0.576,1.169)$ & 06 & 0.504 & 0.552 & 0.610 & .142 & .0210 \\
\hline .05 & 1.75 & $(.226,0.573,1.243)$ & 30.15 & 0.578 & 0.634 & 0.697 & .149 & .0257 \\
\hline .05 & 1.5 & $(.206,0.581,1.371)$ & 28.04 & 0.678 & 0.745 & 0.813 & .157 & .0326 \\
\hline .05 & 1.1 & $(.179,0.674,1.935)$ & 24.20 & 0.951 & 1.036 & 1.108 & .171 & .0533 \\
\hline .05 & 0.9 & $(.185,0.909,3.038)$ & 21.95 & 1.206 & 1.292 & 1.354 & .179 & .0737 \\
\hline .005 & 0.9 & $(.262,0.691,1.480)$ & 8.02 & 1.316 & 1.326 & 1.354 & .302 & .0724 \\
\hline .25 & 0.9 & $(.307,1.369,6.658)$ & 39.81 & 1.017 & 1.292 & 1.354 & .083 & .0816 \\
\hline
\end{tabular}

Rate ${ }^{*}$ of Drift
at $y^{*}$

is the expected rate of change in wealth evaluated at $y=y^{\star}$.

Table 2

$\alpha=0, r_{f}=.01, \delta=0.02, \mu=.059, \quad \sigma=.22$ 


\section{Table 3}

$\delta=0.04, \quad r_{f}=0.01, \alpha=0.00, \mu=0.059, \quad \sigma=0.22$

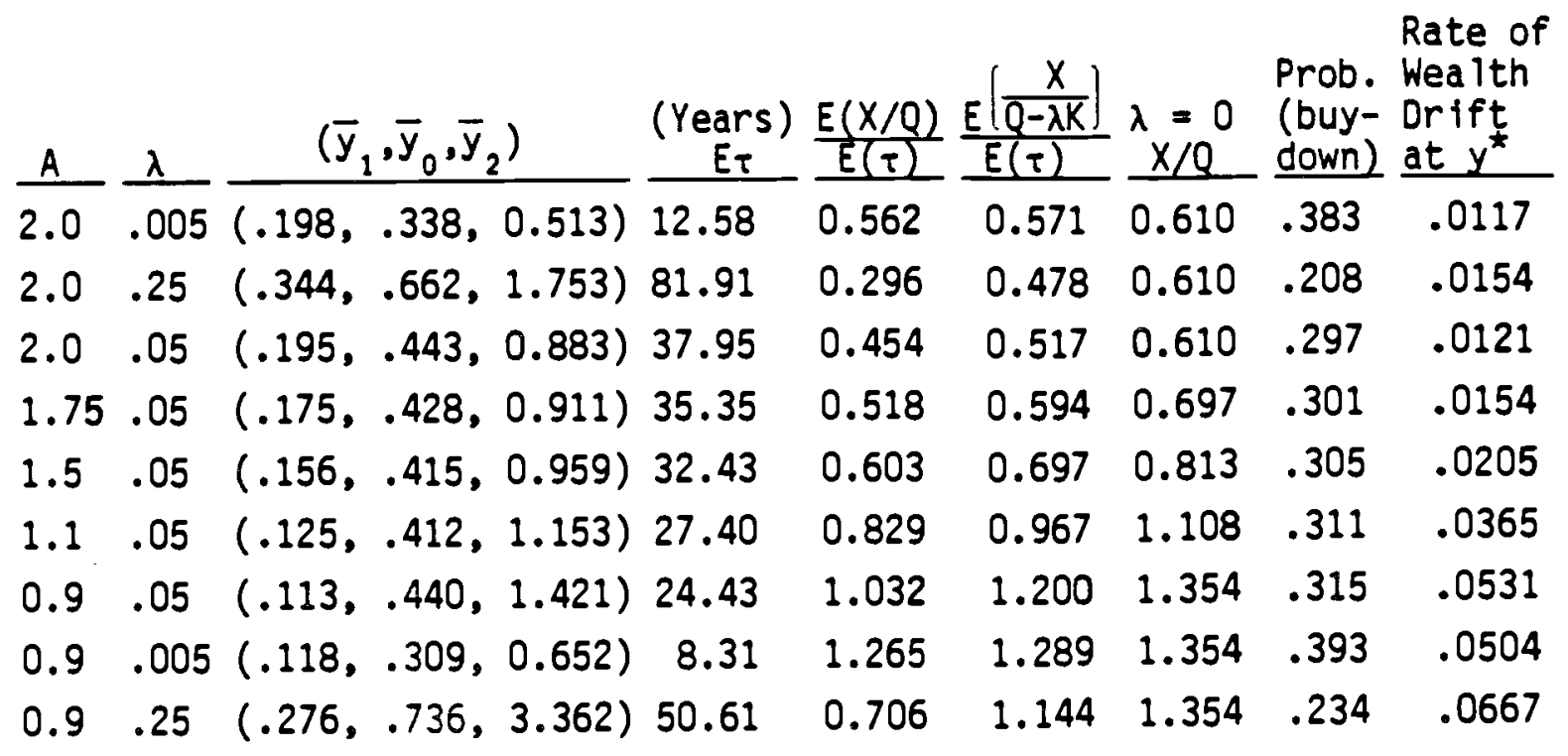

Table 4

$\delta=0.06, \quad r_{f}=0.01, \quad \alpha=0.00, \mu=0.059, \quad \sigma=0.22$

\begin{tabular}{|c|c|c|c|c|c|c|c|c|}
\hline A & $\lambda$ & $\left(\bar{y}_{1}, \bar{y}_{0}, \bar{y}_{2}\right)$ & ET & $\frac{E(X / Q)}{E(\tau)}$ & $\frac{\left(\frac{x}{Q-\lambda K}\right)}{E(\tau)}$ & $\begin{array}{l}\lambda=0 \\
x / 0\end{array}$ & $\begin{array}{l}\text { Prob. } \\
\text { (buy- } \\
\text { down) } \\
\end{array}$ & $\begin{array}{l}\text { If ift } \\
\text { It } y^{*}\end{array}$ \\
\hline & .005 & $6, .268,0$. & 90 & 0.549 & 0.560 & 0.610 & .50 & .00 \\
\hline & 5 & 0,1 & & 0 . & .421 & & .5 & .0076 \\
\hline & .05 & $5, .369,0$ & & & 490 & & .5 & 0042 \\
\hline 75 & .05 & 9. & & 0.468 & 0.561 & 0.697 & .502 & .00 \\
\hline & .05 & 8 & & 0. & 0.657 & 0.813 & .49 & \\
\hline & .05 & $, .314,0$ & 28.77 & 0.727 & 0.906 & 1.108 & .48 & \\
\hline & 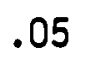 & $?, .312$, & & 0.8 & 1.118 & 54 & .480 & .0 \\
\hline & .0 & 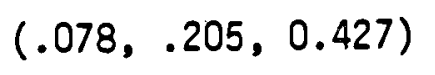 & & & 1.253 & 54 & 488 & .02 \\
\hline & 25 & $. .476)$ & 55.48 & 0. & 1.006 & 1.354 & .469 & .0500 \\
\hline
\end{tabular}




\section{Table 5}

$\mu=0.059, \quad A=1.1, \delta=0.01, \quad \sigma=0.22, \quad r_{f}=0.01, \lambda=0.05$

\begin{tabular}{|c|c|c|c|c|c|c|c|}
\hline$\alpha$ & $\left(\bar{y}_{1}, \bar{y}_{0}, \bar{y}_{2}\right)$ & $\begin{array}{c}\text { (Years) } \\
E_{\tau}\end{array}$ & $\frac{E(X / 0)}{E(\tau)}$ & $\frac{E\left(\frac{X}{0-\lambda K}\right)}{E(\tau)}$ & $\begin{array}{l}\lambda=0 \\
x / 0\end{array}$ & $\begin{array}{l}\text { Prob. } \\
\text { (buy- } \\
\text { down) }\end{array}$ & $\begin{array}{l}\text { Rate of } \\
\text { Wealth } \\
\text { Drift } \\
\text { at } y^{*} \\
\end{array}$ \\
\hline .00 & $(0.26,1.08,3.16)$ & 22.39 & 1.015 & 1.069 & 1.108 & .121 & .0622 \\
\hline .01 & $(0.54,1.86,4.90)$ & 16.32 & 1.048 & 1.078 & 1.108 & .114 & .0714 \\
\hline .02 & $(0.84,2.59,6.46)$ & 13.26 & 1.063 & 1.083 & 1.108 & .099 & .0811 \\
\hline 03 & $(1.14,3.28,7.93)$ & 11.34 & 1.071 & 1.087 & 1.108 & .084 & .0908 \\
\hline .04 & $(1.46,3.94,9.32)$ & 9.92 & 1.077 & 1.090 & 1.108 & .071 & .1006 \\
\hline .05 & $(1.77,4.60,10.67)$ & 8.84 & 1.082 & 1.093 & 1.108 & .059 & .1105 \\
\hline .06 & $(2.08,5.23,12.01)$ & 8.00 & 1.085 & 1.095 & 1.108 & .049 & .1203 \\
\hline .07 & $(2.40,5.85,13.29)$ & 7.28 & 1.088 & 1.096 & 1.108 & .041 & .1302 \\
\hline .08 & $(2.73,6.47,14.55)$ & 6.66 & 1.090 & 1.098 & 1.108 & .034 & .140 \\
\hline .09 & $(3.06,7.07,15.83)$ & 6.16 & 1.092 & 1.099 & 1.108 & .029 & .150 \\
\hline .10 & $(3.37,7.67,17.09$ & 5.73 & 1.094 & 1.100 & 1.108 & .023 & .160 \\
\hline
\end{tabular}




\section{CONCLUSIONS}

In the model, it is optimal for consumers to have a target level $y^{\star}$ for the ratio $y=(Q-\lambda K) / K$ of liquid wealth to durable size. Further, there are two numbers $y_{1}$ and $y_{2}$ such that only if $y \leq y_{1}$ or $y \geq y_{2}$, will the consumer sell his current durable $k$ to return $y$ to the level $y^{\star}$, by purchasing a new durable $K^{\star}(Q)$ which satisfies $y^{\star}=Q / K^{\star}(Q)-\lambda$.

An important implication of the model is that the new size of the durable purchased depends only on wealth. Therefore, to the extent that wealth changes are unpredictable, changes in the size of new durable purchases will be unpredictable. This was pointed out by Bar-I lan and Blinder (1986), who used this idea to test an 111 iquid durable goods model against the standard "permanent income" (1.e. no transactions cost) model of consumption which had been tested by Hall (1978) and Flavin (1981). Z They noted that the average size of the new car purchased at date $t$ is a proxy for $K^{\star}(Q)$ and that changes in this variable should be unpredictable. Indeed they find this correct for automobile purchases. They also note that when one looks at the number of new cars purchased, then changes in this variable should be predictable. Our model cannot be directly applifed to evaluate such a statement without modelling the aggregation over consumers. However, it seems reasonable to conjecture that the number of consumers who arrive at a boundary at time $t$ will depend on the level of durables they each hold at time $t$, and this in turn will depend on the history of prior durable purchases. Therefore, changes in the number of people purchasing new automoblles should be forecastable using information on the prior levels of purchases. They indeed find this to be the case. Therefore, by distinguishing the number of people making new purchases from the average size of each purchase, it appears to be posstble to test predictions of this 
model which are absent from the standard model as well as from the quadratic adjustment cost model of the type studied by Bernanke (1985) and the convex adjustment cost model of Eichenbaum and Hansen (1985). Clearly, further work needs to be done on the aggregation problem before such tests can be made precise. 8

Another area in which further work is needed is in modelling the problems associated with multiple types of consumption goods, each of which has a possibly different transactions cost. In assuming one type of consumption good we ignore the issue of substitution between low transactions cost and high transactions cost goods, and in particular that such substitution might lead a change in wealth to cause an excessive movement in the purchases of low transactions cost goods.

We also ignore the possibllity that there are various types of durable goods which are purchased at staggered dates by a given consumer. In such a situation the consumer may be purchasing some durable good almost every month. It would be interesting to know the correlation between consumption service flows and stock returns in such a mode1.

It should be recognized that many goods which our national income accounts consider to be nondurables, actually have a large "durable" component. This is obvious for categories such as clothing and shoes. However, many nondurables are used in almost fixed proportions with durables, and this creates a transaction cost not dissimilar to that studied here. For example, changing the level of food consumption may have transactions cost, if for example, one has to find different friends with whom to go to better restaurants, or learn about better foods to buy in grocery stores. (Some people even live in areas for which eating better food would require them to change jobs and move out of town.) Changing the level of gasoline, or 
electricity might require selling an automobile, home, or changing a living or work location.

We have shown that small costs of changing consumption levels can lead consumption to be insensitive to wealth for long periods. This makes the consumption based asset pricing mode 1 inappropriate for predicting asset risk premia. However, we show that market portfolio (i.e. wealth) based measures of risk premia continue to be appropriate even in the presence of consumption transactions costs. 


\section{Footnotes}

1. The CCAPM was rejected in tests performed in Hansen and Singleton [1982 and 1983]. Mankiw and Shaptro [1984] compare the adequacy of the CCAPM with the CAPM and find that the later performs better. The most favorable evidence for the CCAPM appears in Breeden, Gibbons and Litzenberger [1986], where the unconditional form of the CCAPM is evaluated.

2. These observations are based upon Grossman, Melino and Shiller [1985].

3. See Constantinides[1986] for results regarding proportional transaction costs in security trading and a survey of work on securfty transaction costs. See Harrison and Taylor [1978], and Richard [1977] for related work on the optimal stopping of a controlled diffusion.

4. To see this, note that in any neighborhood of $(Q, K)$ in which $V$ is twice continuously differentiable and no stopping occurs, the Bellman equation is

$$
\begin{gathered}
0=\sup _{X}\left\{u(K)-\delta V(Q, K)+\left(\underline{X} \cdot \underline{u}+r_{f}(Q-K)-\alpha K\right) V_{1}(Q, K)+\frac{1}{2} v_{11}(Q, K) \underline{x} \cdot \tilde{E} \cdot \underline{x}+\right. \\
\left.v_{2}(Q, K) \alpha K\right\} .
\end{gathered}
$$

Clearly $V_{1} \geq 0$. If $V_{1}=0$, then this equation can only hold if $v_{11}=0$. In such a case any value of $x$ is optimal. If $v_{1}>0$, then the equation requires that $v_{11}<0$ and the optimal portfolio satisfies $\underline{x}=\frac{-v_{1}}{v_{11}} \Sigma^{-1} \cdot \underline{u}$.

5. Economic Report of the President 1980, pp.260-261. See Stambaugh [1982] for a discussion of real returns on various durables and assets.

6. This is analogous to what would occur in a certainty mode with $r>\delta$. There, both consumption and wealth would "drift" upward at the same rate. 
7. Bar-I lan and Blinder analyzed a model without uncertainty, but their insights can be directly applied to the model of this paper.

8 Caplin and Spulber (1985) consider a model with fixed transactions costs where aggregation over consumers causes the model to behave in many ways like a representative agent model without transactions cost. We do not expect a similar result here because starting from any cross-sectional distribution of consumer state variables, at time 0 , the cross-sectiona 1 distribution at time $t$ (and thus the density of house purchases) will depend on the history of the stock market between 0 and $t$. In the Caplin and Spulber model this is not the case because random shocks are a lways of the same sign so it is as if the cross-sectional distribution of characteristics revolves in one direction around a circle; always maintaining the same density at each point.

9) To extend the analys is to the case of several assets, take

$$
\begin{aligned}
& \underline{\varepsilon}=\varepsilon \Sigma^{-1} \cdot \underline{\mu} \\
& \underline{x}^{h}=x^{h} \Sigma^{-1} \cdot \underline{\mu} .
\end{aligned}
$$

The argument of Footnote 4, transposed to the proof of Lemma 2, shows that the optimal portfolio is proportional to $\sum^{-1} \mu$. 


\section{REFERENCES}

Bar-Ilan, A., and A. Blinder, "The Life-Cycle Permanent-Income Model and Consumer Durables," mimeo May 1986.

Bernanke, B., "Adjustment Costs, Durables and Aggregate Consumption," Journa 1 of Monetary Economics, 1985, 13, p.41-68.

Breeden, D., M. Gibbons, and R. Litzenberger, "Empirical Tests of the Consumption Oriented CAPM," Stanford Graduate School of Business Research Paper No. 879, March 1986.

Caplin, A., and D. Spulber, "Inflation Menu Costs and Endogenous Price Variability," mimeo, August 1985.

Constant infdes, G., "Capital Market Equilibrium with Transaction Costs," Journal of Political Economy, Vo1. 94, No. 4, Aug. 1986, pp.842-62.

Eichenbaum, M. and L. Hansen, "Estimating Models with Intertemporal Substitution Using Aggregate Time Series Data," manuscript, CarnegieMellon Untversity, 1985.

Flavin, M.A., "The Adjustment of Consumption to Changing Expectations about Future Income," Journal of Polftical Economy, 1981, 89, pp.971-10009.

Flemming, J.S., "The Utility of Wealth and the Utility of Windfalls," Review of Economic Studies, 1969,36 , pp.55-66.

Grossman, S., A. Melino, and R. Shiller, "Estimating the Continuous Time Consumption Based Asset Pricing Model," National Bureau of Economic Research Working Paper No. 1643, June 1985.

Ha11, R.E., "Stochastic Implication of the Life Cycle-Permanent Income Hypothes is: Theory and Evidence," Journal of Political Economy, 1978, 86, pp.971-87.

Hansen, L.P., and K.J. Singleton, "Generalized Instrumental Variables Estimation of Noni inear Rational Expectations Mode1s, "Econometrica 1982, 50 , pp.1269-82.

Hansen, L.P., and K.J. Singleton, "Stochastic Consumption, Risk Aversion and the Temporal Behavior of Stock Returns, Journal of Political Economy, 1983.

Harrison, J., and A. Taylor, "Optimal Control of a Brownian Motion Storage System," Stochastic Processes and Their Applications, Vol. 6, No. 2, Jan. 1978, pp.179-94.

Ibbotson, R., and R. Sinquefeld, Stocks, Bonds, B11ls and Inflation: The Past and the Future, 1982 Edition, Financial Research Analyst's Foundation (Charlottesville: 1982). 
Karatzas, I., J.P. Lehoczky, S.P. Sethi, and S.E. Shreve, "Explicit Solution of a General Consumption/Investment Problem," Math of Operations Research, Vo1. 11, No. 2, May 1986, pp. 261-94.

Karlin, S., and H. Taylor, A Second Course in Stochastic Processes, Academic Press (New York: 1981).

Krylov, N.V., Controlled Diffus ion Processes, Springer-Verlag (New York: 1980).

Mank iw, N.G., and M. Shapiro, "Risk and Return: Consumption Versus Market Beta," National Buearu of Economic Research Working Paper No. 1399, July 1984.

Merton, R., "Lifetime Portfolio Selection under Uncertainty: The Continuous Time Case," Rev. Econ and Stat., Vo1. 51, 1969, pp.247-57.

Richard, S., "Optimal Impulse Control of a Diffusion Process with Both Fixed and Proportional Costs of Control," SIAM J. Control and Optimization, Vol. 15, No. 1, Jan 77, pp.79-91.

Stambaugh, R., "On Exclusion of Assets from Tests of the Two-Parameter Mode1: A Sensitivity Analys is," Journal of Financial Economics, Vol. 10, No. 3, Nov. 1982, pp.237-68. 


\section{APPENDIX}

Most of the Appendix deals with the optimization problem (2.9) - (2.12) of Section 2. However, as a preliminary step we prove Theorem 2.1 concerning the value function $V(Q, K)$. Further, to simplify notations, we consider the case of a single risky asset with drift $\mu+r_{f}$ and instantaneous variance $\sigma^{2}$ (we indicate in footnotes how to extend the analys is to the case of several assets, where appropriate).

\section{Proof of theorem 2.1:}

1) It is always possible for the agent to sell immediately his current durable good, and to invest all his wealth $Q-\lambda K$ into a house to be kept for all $t \geqslant 0$, without undertaking any financial operation in the future $\left(Q_{t}=K_{t}, X_{t}=0\right.$ for all $\left.t \geqslant 0\right)$. If $a>0$, this gives:

$$
V(Q, K) \geqslant \frac{(Q-\lambda K)^{a}}{a(\delta+a \alpha)}
$$

and $v_{2} \equiv(\delta+a \alpha)^{-1}$. If $a<0$, then consider the strategy of setting $x_{t} \equiv 0$ and buying a new house every year. If $r>0$ and $\lambda<1$ then this strategy will do at least as well as when $r=0$ and $\lambda=1$. Thus, assume that $Q-\lambda K$ is moved to an economy where henceforth $r=0$ and $\lambda=1$. In year $n$ a fraction $g_{n}$ of $Q-\lambda K$ is invested in housing, where

$$
g_{n}=e^{\frac{\delta n}{2 a}}\left(1-e^{\frac{\delta}{2 a}}\right) \text {. }
$$

It is easily verified that $g_{n} \in(0,1)$ and $\sum_{n=0}^{\infty} g_{n}=1$, since $a<0$.

It follows that setting $K(n)=(Q-\lambda K) g_{n}$ is feasible and yields a discounted utility of

$$
\sum_{n=0}^{\infty} \int_{n}^{n+1} e^{-\delta t} u\left(g_{n}(Q-\lambda K) e^{-\alpha(t-n)}\right) d t
$$




$$
\begin{gathered}
=\frac{(Q-\lambda K)^{a}}{a} v_{2}, \text { where } \\
v_{2} \equiv \frac{\left(1-e^{\frac{\delta}{2 a}}\right)^{a}}{1-e^{-\frac{\delta}{2}}} .
\end{gathered}
$$

(2) When $\lambda=0, V$ does not depend on $K$, and $Q$ becomes the only state variable, while $K$ and $X$ are control varlables. It is thus identical to the standard model of consumption and portfolio choice as in Merton (1969). Since (2.1) and (2.3) are linear in $(Q, K, X)$ and since

$$
V(Q)=E \int_{0}^{\infty} e^{-\delta t} \frac{K_{t}^{a}}{a} d t,
$$

the solution, if it exists, must be, by homogeneity, of the form $V(Q)=v Q^{a}$, where $v=V(1)$. This model has been studied by Karatzas, et.a1. [1986, p.290] who show that (our) $B>0$ is sufficient to ensure that $\nu$ is finite, where it is crucial to note that we require $Q_{t} \geqslant 0$, and if $Q_{t}=0$, then the process is stopped and the consumer gets $u(0)$. The Beliman differential equation is

(A. 1) $\sup _{(K, X)}\left[\frac{\sigma^{2} X^{2}}{2} V^{\prime \prime}(Q)+\left(r_{F} Q-\left(r_{F}+a\right) K+\mu X\right) V^{\prime}(Q)-\delta V(Q)+\frac{K^{a}}{a}\right]=0$. Substituting $V(Q)=\nu Q^{a}$ into (A.1) yields optimal solutions

$$
X=\frac{\mu Q}{\sigma^{2}(1-a)} \quad K=\left[a\left(r_{F}+\alpha\right) \nu\right]^{\frac{1}{a-1}} Q \text {, }
$$

and a formula for v: 
(A.2) $\quad\left(r_{F}+\alpha\right)(1-a)\left[a\left(r_{F}+\alpha\right) \nu\right]^{\frac{1}{a-1}}=\beta$.

If $B>0$, then (A.2) can be solved for $v$, and thus there is a solution to $(A .1)$.

Since the consumer is obviously better off when $\lambda=0$ than when $\lambda>0$, it follows that $v Q^{\mathrm{a}} \geqslant V(Q, K)$. Q.E.D.

The rest of the Appendix solves the optimization problem (2.9) - (2.12). Given the state (or our knowledge...) of the mathematical 1iterature, we have been lead to take a rather roundabout approach.

First for fixed $M$, the optimization problem in (2.9) - (2.11) is of the form studied by Krylov (1980). There are however two important differences. Krylov assumes that $(i) x_{t}$ is bounded and bounded away from zero, 1.e., there are two numbers $x^{h}>\varepsilon>0$, with $x^{h} \geqslant x_{t} \geqslant \varepsilon$, and (1i) $y_{t}$ belongs to a bounded state space, in the sense that there are numbers $y^{l}, y^{h}, y^{l}<y^{h}$, such that if $y_{t}=y^{l}$ or if $y_{t}=y^{h}$, then the consumer is forced to stop (i.e. set $\tau=0$ ). To solve the problem, we proceed as follows. We define an auxiliary problem, with artificlal bounds, which satisfies the assumptions of Krylov, and we let $x^{h}$ go to infinity and $\varepsilon$ go to zero. Lemma 1, based on a result of Krylov, shows that this procedure is justified: the value of the auxiliary problem converges uniformly to the value of the limit problem. Lemmas 2, 3 and 4 characterize the solution and the optimal strategy of the auxiliary problem. Remark 1 shows that for $x^{h}$ large enough the upper bound in ( $i$ ) is not binding. The first part of the proof is concluded by Lemma 5 , which describes the solution of the optimization problem (2.9) - (2.11) for fixed $M$ under the assumption (ii), by taking the limit of the optimal strategies of the auxiliary problems. 
The second part of the proof uses (2.12) to find M. Lemmas 6 and 7 show how the values obtained at the end of the first part vary with $M$ and $\lambda$, and Lemma 8 characterizes the solution of the problem under the constraint (ii) $y^{l} \leqslant y_{t} \leqslant y^{h}$. It is only at this late stage in Lemma 9, that we are able to show that, for $y^{\ell}$ small enough and $y^{h}$ large enough, (ii) is not binding so that we can dispense from it.

We first study an auxiliary problem where the following constraints are added to the optimization in (2.9) - (2.10), $M$ is a real number of the same sign as $a$, and $h_{\varepsilon}(y ; M)$ denotes the maximized value of the objective. 9

$$
x^{h} \geqslant x_{t} \geqslant \varepsilon>0 \quad \text { for all } t
$$

$$
y^{\ell} \leqslant y_{t} \leqslant y^{h} \text { for all } t
$$

with $y^{\ell}>0$, and

$$
\begin{array}{lll}
y_{t}=y^{h} & \text { implies } & h_{\varepsilon}\left(y_{t} ; M\right)=M y_{t}^{a} \\
y_{t}=y^{l} & \text { implies } & h_{\varepsilon}\left(y_{t} ; M\right)=M y_{t}^{a} .
\end{array}
$$

Note that $h_{\varepsilon}(y ; M)$ a priorf depends on $\left(\varepsilon, x^{h}, y^{l}, y^{h}\right)$. We have only made explicit its dependency in $\varepsilon$. We let $h(y ; M)$ denote the solution of the auxiliary problem with $\varepsilon$ equal to zero.

Lemma 1. When $\varepsilon$ converges to zero, the function $h_{\varepsilon}(y ; M)$ converges uniformly to $h(y ; M)$ on $\left[y^{\ell}, y^{h}\right]$ and $h(y ; M)$ is continuous in $M$.

Proof of Lemma 1. The assumptions of Krylov [1980, p. 130] are satisfied. We apply corollary 13, p. 138. Corollary 13 applies in fact to:

$$
h_{\varepsilon}(y, T ; M) \equiv \sup _{\tau \leq T,\left(x_{t}\right)} E\left[\int_{0}^{\tau} \frac{e^{-\bar{\delta} t}}{a} d t+e^{-\bar{\delta} \tau} M y_{\tau}^{a}\right]
$$


and states that $h_{\varepsilon}(y, T ; M)$ converges uniformly to $h(y, T ; M)$, and that both are continuous in $\mathrm{M}$. We have

$$
\begin{aligned}
& \left|h_{\varepsilon}(y ; M)-h_{\varepsilon}(y, T ; M)\right| \leq e^{-\bar{\delta} T} \sup _{y}\left|h_{\varepsilon}(y ; M)\right| \leq e^{-\bar{\delta} T} \sup _{y} \mid M(y+\lambda)^{a_{1}} \\
& |h(y ; M)-h(y, T ; M)| \leq e^{-\bar{\delta} T} \sup _{y}|h(y ; M)| \leq e^{-\bar{\delta} T} \sup _{y} \mid M(y+\lambda)^{a_{1}}
\end{aligned}
$$

and the difference can be made as small as one wishes for $T$ large enough given $y^{\ell}, y^{h}$. Now:

$$
\begin{aligned}
\left|h(y ; M)-h_{\varepsilon}(y ; M)\right| \leq & h(y ; M)-h(y, T ; M) 1+ \\
& \mid h(y, T ; M)-h_{\varepsilon}(y, T ; M) 1+ \\
& i h_{\varepsilon}(y, T ; M)-h_{\varepsilon}(y: M) 1 .
\end{aligned}
$$

The first and third terms go (uniformly) to zero by the preceding inequalities while the second term converges unfformly to zero by corollary 13 of Krylov.

We now proceed to study the auxiliary problem.

Lemma 2: $\quad h_{\varepsilon}(y ; M)$ is continuous ly differentiable in $y$ on $\left[y^{l}, y^{h}\right]$. For $x^{h}, y^{h}$ large enough, $\varepsilon$ and $y^{\ell}$ small enough, for $M<(1-\lambda)^{-a} v$ there exists an interval $\left(y_{1}^{\varepsilon}, y_{2}^{\varepsilon}\right)$ with $y_{1}^{\varepsilon}<y_{2}^{\varepsilon}$, such that:

(i) $h_{\varepsilon}(y ; M)$ is twice continuous ly differentiable and $h_{\varepsilon}(y ; M)>M y^{a}$ on $\left(y_{1}^{\varepsilon}, y_{2}^{\varepsilon}\right) ; \bar{y}=(1-\lambda)(1-a) r / \beta$ belongs to $\left(y_{1}^{\varepsilon}, y_{2}^{\varepsilon}\right)$.

(ii) $h_{\varepsilon}(y ; M)=M y^{a}$ on the complement of $\left(y_{1}^{\varepsilon}, y_{2}^{\varepsilon}\right)$ in $\left[y^{l}, y^{h}\right]$. For $M>(1-\lambda)^{-a} v, h_{\varepsilon}(y ; M)=M y^{a}$ on $\left[y^{l}, y^{h}\right]$.

Proof of Lemma 2: It is immediate to check that the auxiliary problem satisfies the assumptions of Krylov [1980, p. 22]. Therefore, by Krylov's Theorem 3, $h_{\varepsilon}$ is a continuously differentiable function of $y$ for $y \in\left[y^{\ell}, y^{h}\right]$, and dropping the arguments of the function $h_{\varepsilon}$ while 
using $h_{\varepsilon}^{\prime}$ and $h_{\varepsilon}^{\prime \prime}$ for the first and second derivatives with respect to $y$, we have on the set $C \equiv\left\{y: h_{\varepsilon}(y ; M)>M y^{a}\right\}$ :

(A.5) $\operatorname{Sup}_{x}\left[\frac{x^{2}}{2} \sigma^{2} h_{\varepsilon}^{\prime \prime}+(r y+x \mu-r(1-\lambda)) h_{\varepsilon}^{\prime}-\bar{\delta} h_{\varepsilon}+\frac{1}{a}\right]=0$,

which can be rewritten as:

$$
\operatorname{Sup}_{x}\left\{\frac{\sigma^{2} h_{\varepsilon}^{\prime \prime}}{2}\left[\left(x+\frac{\mu h_{\varepsilon}^{\prime}}{\sigma^{2} h_{\varepsilon}^{\prime \prime}}\right]^{2}-\left[\frac{\mu h_{\varepsilon}^{\prime}}{\sigma^{2} h_{\varepsilon}^{\prime \prime}}\right]^{2}\right]+r(y+\lambda-1) h_{\varepsilon}^{\prime}-\bar{\delta} h_{\varepsilon}+\frac{1}{a}\right\}=0 .
$$

Assuming $x^{h}$ is large, and $x$ does not hit $x^{h}$, we get

if $-\frac{\mu h_{\varepsilon}^{\prime}}{\sigma^{2} h_{\varepsilon}^{\prime \prime}} \geqslant \varepsilon$ then:

$(A .6)$

$$
x(y)=-\frac{\mu h_{\varepsilon}^{\prime}}{\sigma^{2} h_{\varepsilon}^{\prime \prime}} \text { and }
$$

(A.7) $\quad-\frac{1}{2} \frac{\mu^{2}}{\sigma^{2}} \frac{h_{\varepsilon}^{\prime 2}}{h_{\varepsilon}^{\prime \prime}}+r(y+\lambda-1) h_{\varepsilon}^{\prime}-\bar{\delta} h_{\varepsilon}+\frac{1}{a}=0 ;$

if $\quad-\frac{\mu h_{\varepsilon}^{\prime}}{\sigma^{2} h_{\varepsilon}^{\prime \prime}}<\varepsilon$ then $x(y)=\varepsilon$, and

$(A .8)$

$$
\frac{\varepsilon^{2}}{2} \sigma^{2} h_{\varepsilon}^{\prime \prime}+(r(y+\lambda-1)+\mu \varepsilon) h_{\varepsilon}^{\prime}-\bar{\delta} h_{\varepsilon}+\frac{1}{a}=0,
$$

and

(A.9) $\quad-\frac{1}{2} \frac{\mu^{2}}{\sigma^{2}} \frac{h_{\varepsilon}^{\prime 2}}{h_{\varepsilon}^{11}}+r(y+\lambda-1) h_{\varepsilon}^{\prime}-\bar{\delta} h_{\varepsilon}+\frac{1}{a}>0$.

Note that (A.7) can be rewritten as:

6 
(A.10) $\quad G\left(h_{\varepsilon}^{\prime \prime}, h_{\varepsilon}^{\prime}, h_{\varepsilon}, y\right)=0$

where $G$ is increasing in $h_{\varepsilon}^{\prime \prime}$ and strfctly decreasing in $h_{\varepsilon}$.

Let

(A.11) $g(y)=G\left[a(a-1) M y^{a-2}, a M y^{a-1}, M y^{a}, y\right]$

$$
g(y)=-M B y^{a}-(1-\lambda) a M r y^{a-1}+\frac{1}{a} .
$$

We have:

$$
g^{\prime}(y)=-a M y^{a-2}[\beta y-(1-\lambda)(1-a) r] .
$$

Since $a M>0$, by (2.13), $g(y)$ is increasing for $0 \leqslant y \leqslant \bar{y}$

where $\bar{y}=(1-\lambda)(1-a) r / \beta$ and decreasing for $\bar{y} \leqslant y$.

$$
\begin{aligned}
& g(\bar{y})=-M \bar{y}^{-a-1}[(1-\lambda)(1-a) r+(1-\lambda) a r]+\frac{1}{a} \\
& g(\bar{y})=-M \bar{y}^{-a-1}(1-\lambda) r+\frac{1}{a}=\bar{y}^{-a-1}(1-\lambda) r\left[v(1-\lambda)^{-a}-M\right] .
\end{aligned}
$$

Therefore, by (2.13), $g(\bar{y})$ is strictly positive for $M<(1-\lambda)^{-a} v$.

The proof of the lemma uses the following remark: if $y \geqslant y_{\varepsilon} \equiv \sigma^{2}(1-a) \varepsilon / \mu$ and $h_{\varepsilon}(y)=M y^{a}$, then $g(y) \leqslant 0$. The remark is true because $h_{\varepsilon}(z) \geqslant M z^{a}$ all $z$, thus for $y \geqslant h_{\varepsilon}(y)=M y^{a}$ we have

$$
h_{\varepsilon}^{\prime}(y)=a M y^{a-1} \text { and } h_{\varepsilon}^{\prime \prime}(y) \geqslant a(a-1) M y^{a-2} \text {, }
$$

where $h_{\varepsilon}^{\prime \prime}\left(y_{\varepsilon}\right)$ refers to the derivative computed from the interior of the set $\left\{y: h_{\varepsilon}(y ; M)>M y^{a}\right\}$ in case $y_{\varepsilon}$ is a boundary point of that set.

Therefore,

$$
-\frac{\mu h_{\varepsilon}^{\prime}(y)}{\sigma^{2} h_{\varepsilon}^{\prime \prime}(y)} \geqslant \frac{\mu}{\sigma^{2}} \frac{y}{(1-a)} \geqslant \frac{\mu}{\sigma^{2}} \frac{y_{\varepsilon}}{1-a}=\varepsilon
$$

so that (A.7) holds, which implies, using (A.10) and (A.11), that $g(y) \leqslant 0$. 
Since $g(\bar{y})>0$ and $\varepsilon$ can be chosen sufficiently small that $\bar{y}>y_{\varepsilon}$, the preceding remark implies $h_{\varepsilon}(\bar{y} ; M)>M \bar{y}^{a}$. That is, $\left(y^{\ell}, y^{h}\right)$ can be chosen to include $\bar{y}$. Let $\left(y_{1}^{\varepsilon}, y_{2}^{\varepsilon}\right)$ be the largest interval containing $\bar{y}$ such that $h_{\varepsilon}(y ; M)>M y^{a}$. By the foregoing remark, we have

$y_{1}^{\varepsilon} \geqslant y_{\varepsilon}$ implies $h_{\varepsilon}\left(y_{1}^{\varepsilon} ; M\right)=M y_{1}^{a}$ and $g\left(y_{1}^{\varepsilon}\right)<0$

$$
h_{\varepsilon}\left(y_{2}^{\varepsilon} ; M\right)=M y_{2}^{a} \text { and } g\left(y_{2}^{\varepsilon}\right) \leqslant 0 \text {. }
$$

Now the shape of the function $g(y)$ implies that $g(y)<0$ for $y<y_{1}^{\varepsilon}$ and $y>y_{2}^{\varepsilon}$ provided we choose $y_{\varepsilon}$ small enough so that $g\left(y_{\varepsilon}\right)<0$. (Note that $g(y) \rightarrow-\infty$ as $y \rightarrow 0$.) We use this fact to prove part (ii) of the lemma by contradiction. Suppose there was another interval $\left(y_{1}^{\prime}, y_{2}^{\prime}\right)$ disjoint from $\left(y_{1}^{\varepsilon}, y_{2}^{\varepsilon}\right)$ such that $h_{\varepsilon}(y)>M y^{a}$ for $y$ in $\left(y_{1}^{\prime}, y_{2}^{\prime}\right)$. Note that $h(y)=M y^{a}$ at $y=y^{\ell}$ and $y=y^{h}$. Therefore, there is at least a point $y^{\prime}$ in $\left(y_{1}^{\prime}, y_{2}^{\prime}\right)$ which maximizes $h_{\varepsilon}(y ; M)-M y^{a}$ on $\left[y_{1}^{\prime}, y_{2}^{\prime}\right]$. At $y^{\prime}$ :

$$
\begin{aligned}
& h_{\varepsilon}\left(y^{\prime} ; M\right)>M y^{\prime a} \\
& h_{\varepsilon}^{\prime}\left(y^{\prime} ; M\right)=a M y^{\prime a-1} \\
& h_{\varepsilon}^{\prime \prime}\left(y^{\prime} ; M\right) \leqslant a(a-1) M y^{\prime a-2} .
\end{aligned}
$$

It follows that $g\left(y^{\prime}\right)>0$, since either (A.7) or (A.9) holds at $y^{\prime}$, and this contradicts the fact that $g(y)<0$ on the complement of $\left(y_{1}^{\varepsilon}, y_{2}^{\varepsilon}\right)$. Finally, for $M>(1-\lambda)^{-a} v, g(y)$ is negative for all y. If $\left(y_{1}^{\varepsilon}, y_{2}^{\varepsilon}\right)$ was not empty, the maximizer $y^{\prime}$ of $h_{\varepsilon}(y ; M)-M y^{a}$ on $\left[y_{1}^{\varepsilon}, y_{2}^{\varepsilon}\right]$ would satisfy $g\left(y^{\prime}\right)>0$, a contradiction.

It will be very useful to get a precise description of the shape of the optimal policy $x(y)$ on $\left(y_{1}^{\varepsilon}, y_{2}^{\varepsilon}\right)$. This involves a careful analys is 
of the differential equation (3.11). The following Lemma uses the notation of (3.12)-(3.14).

Lemma 3: a solution of (3.11) has one of the three possible following shapes in the positive orthant:

(1) $0 \leq x(y) \leq \theta_{1}(y+\lambda-1), x^{\prime}(y)<0, x^{\prime \prime}(y) \leq 0$, defined only on a subset of $[0,1-\lambda]$,

(ii) $x(y)>\operatorname{Max}\left(\theta_{1}(y+\lambda-1), \theta_{2}(y+\lambda-1)\right), x^{\prime \prime}(y)>0$,

(iii) $0 \leq x(y) \leq \theta_{2}(y+\lambda-1), x^{\prime}(y)>0, x^{\prime \prime}(y) \leq 0$, defined on a subset of $[1-\lambda,+\infty)$.

Proof of Lemma 3: differentiating (3.11) after dividing through by $x$ gives:

$$
\frac{x^{\prime \prime}}{2}=\frac{r}{\sigma^{2}} \frac{\left(x-(y+\lambda-1) x^{\prime}\right)}{x^{2}} \text {. }
$$

Now:

$$
\frac{x-(y+\lambda-1) x^{\prime}}{x}=1+2\left[\frac{r-\bar{\delta}}{\mu}-\frac{\mu}{2 \sigma^{2}}\right] \frac{y+\lambda-1}{x}-\frac{2 r}{\sigma^{2}}\left(\frac{y+\lambda-1}{x}\right)^{2}
$$

which implies, by definition of $\theta_{1}$ and $\theta_{2}$, that for $x>0$ :

$$
\begin{aligned}
& x^{\prime \prime}<0 \text { for } \theta_{1}<\frac{x}{y+\lambda-1}<\theta_{2} \text {, i.e., } x^{\prime \prime}<0 \text { either when } \\
& \quad x<\theta_{1}(y+\lambda-1) \text {, and } y<1-\lambda, \\
& \text { or when } x>\theta_{2}(y+\lambda-1) \text {, and } y>1-\lambda ; \\
& x^{\prime \prime}>0 \text { for } x>\operatorname{Max}\left[\theta_{1}(y+\lambda-1), \theta_{2}(y+\lambda-1)\right] \text {. }
\end{aligned}
$$

Finally (3.11) gives the sign of $x^{\prime}$. $\quad$ Q.E.D.

Remark 1: The preceding analys is allows to justify that $x^{h}$ can be chosen large enough so that the upper bound is not attained by the optimal strategy. By Krylov [1980, p.39, Theorem 3], it is always true, even if the upper bound is attained, that $h_{\varepsilon}(y ; M)$ is twice continuously differentiable on the set $C$ and the argument at the end of Lemma 2 shows that there is always a point $y^{\prime}$ in $\left[y^{\ell}, y^{h}\right]$ such that: 


$$
-\frac{\mu h_{\varepsilon}^{\prime}}{\sigma^{2} h^{\prime \prime}} \leqslant \frac{\mu y^{\prime}}{\sigma_{\varepsilon}^{2}(1-a)} .
$$

Letting $x^{h}>\operatorname{Max}\left(\varepsilon, \mu y^{h} / \sigma^{2}(1-a)\right)$, this implies that the continuous function $x(y)$ satisfies (A.6) on a nonempty subset of $\left[y^{l}, y^{h}\right]$. The shape of the solution of $(A .6)$, as deduced from Lemma 3 shows then that $x^{h}$ can be taken large enough so as not to be attained. Precisely, in the cases where Lemma $3(i)$ and (iii) are relevant, we can take

$$
x^{h}>\operatorname{Max}\left(\theta_{1}\left(y^{l}+\lambda-1\right), \theta_{2}\left(y^{h}+1-\lambda\right)\right) .
$$

To tackle the situation where $x(y)$ satisfies Lemma 3 (ii) note that by Lemma 2 there is always a point $y^{\prime}$ such that

$$
x\left(y^{\prime}\right) \leqslant \frac{\mu y^{\prime}}{\sigma^{2}(1-a)}
$$

Let $\bar{c}$ be the maximum value of $c$ such that the convex set

$$
\{(x, y))\left(x-\theta_{1}(y+\lambda-1)^{1-\gamma}\left(x-\theta_{2}(y+\lambda-1)^{1+\gamma} \geqslant c\right\}\right.
$$

has a nonempty intersection with the set $\left\{(x, y) \mid x \leq \mu y / \sigma^{2}(1-a)\right\}$. Let $\bar{x}^{-1}$, $i=h, l$ be two values taken by $x$ when $y$ equals $y^{i}, i=h, l$ along the curve

$$
\left(x-\theta_{1}(y+\lambda-1)\right)^{1-\gamma}\left(x-\theta_{2}(y+\lambda-1)\right)^{1+\gamma}=\bar{c} .
$$

To be sure that the upper bound $x^{h}$ is not binding, it suffices then to take

$$
x^{h}>\operatorname{Max}\left(\bar{x}^{l}, \bar{x}^{h}\right) \text {. }
$$

We are now in a position to describe the optimal strategy $x(y)$ associated with the auxiliary problem $\varepsilon$.

Lemma 4: $x(y)$ is continuous on $\left(y_{1}^{\varepsilon}, y_{2}^{\varepsilon}\right)$. Furthermore, for $\varepsilon$ sma11 enough, there exist two positive numbers $z_{1}^{\varepsilon}, z_{2}^{\varepsilon}$ with $y_{1}^{\varepsilon} \leq z_{1}^{\varepsilon} \leq z_{2}^{\varepsilon}<y_{2}^{\varepsilon}$ 


\section{such that:}

(i) $x(y)$ satisfies (3.11) on $\left(y_{1}^{\varepsilon}, z_{1}^{\varepsilon}\right] . x(y)$ satisfies (3.11) on $\left[z_{2}^{\varepsilon}, y_{2}^{\varepsilon}\right)$

(ii) If $z_{1}^{\varepsilon}<z_{2}^{\varepsilon}, x(y)=\varepsilon$ on $\left[z_{1}^{\varepsilon}, z_{2}^{\varepsilon}\right]$. If $z_{1}^{\varepsilon}=z_{2}^{\varepsilon}$, then $x(y)$ satisfies (3.14) on $\left(y_{1}^{\varepsilon}, y_{2}^{\varepsilon}\right)$.

Proof of Lemma 4: When $x(y)$ is larger than $\varepsilon$, we have by substituting $(A .6)$ into $(A .7)$ :

$$
\frac{\mu}{2} x+r(y+\lambda-1)+\frac{1 / a-\bar{\delta} h}{h^{\prime}}=0 \text {. }
$$

Differentiating once, and using (3.8) again, leads to (3.10):

$$
\frac{\mu}{2} x^{\prime} x+\left(r-\bar{\delta}-\frac{\mu^{2}}{2 \sigma^{2}}\right) x-\frac{\mu r}{\sigma^{2}}(y+\lambda-1)=0 \text {. }
$$

Therefore, Lemma 3 gives the shape of $x(y)$ when $x(y) \geq \varepsilon$. Now, by Lemma 2, $\quad h_{y}^{\prime}=M a y^{a-1}$ at $y=y_{1}^{\varepsilon}, y_{2}^{\varepsilon}$, and $h_{\varepsilon}(y ; M) \geq M y^{a}$ implies $h_{y}^{\prime \prime} \geq a(a-1) M y^{a-2}$. Therefore

$$
\lim _{y \rightarrow y \varepsilon_{1}^{\varepsilon}} x(y) \geq \operatorname{Max}\left(\frac{\mu y_{i}^{\varepsilon}}{\sigma^{2}(1-a)}, \varepsilon\right) \text { for } i=1,2 \text {. }
$$

For $\varepsilon$ small enough, $x\left(y_{2}^{\varepsilon}\right)>\varepsilon$, and the shape of the solutions of (3.14) described in Lemma 3 give the desired result.

This completes the description of the solution of the auxiliary problem for $x^{h}=+\infty$ and $\varepsilon>0$. Using Lemma 1 , we study now the function $h(y ; M)$, letting $\varepsilon$ go to zero.

Lemma 5: $h(y ; M)$ is continuously differentiable strictly increasing in $y$ on $\left[y^{\ell}, y^{h}\right]$, except perhaps at $y=1-\lambda$. For $M<(1-\lambda)^{-a_{v}}$, there exists an interval $\left(y_{1}, y_{2}\right)$, such that 
(i) $h(y ; M)>M y^{a}$ on $\left(y_{1}, y_{2}\right)$ and $h(y ; M)$ is twice continuous ly differentiable on $\left(y_{1}, y_{2}\right)$, except perhaps at $y=1-\lambda$. $\bar{y}=(1-\lambda)(1-a) r / B$ belongs to $\left(y_{1}, y_{2}\right)$.

(ii) $h(y ; M)=M y^{a}$ on the complement of $\left(y_{1}, y_{2}\right)$ in $\left[y^{2}, y^{h}\right]$. Moreover, the optimal policy $x(y)$, defined on $\left(y_{1}, y_{2}\right)$, is a continuous function of $y$ such that:

$$
x(y) \geq \operatorname{Max}\left(\theta_{1}(y+\lambda-1), \theta_{2}(y+\lambda-1)\right)
$$

and it satisfies (3.14) for some $c \geq 0$ :

$$
\left(x(y)-\theta_{1}(y+\lambda-1)\right)^{1-\gamma}\left(x(y)-\theta_{2}(y+\lambda-1)\right)^{1+\gamma}=c .
$$

Proof of Lemma 5: first note that for $\varepsilon>\varepsilon^{\prime}, h_{\varepsilon}(y ; M) \leq h_{\varepsilon^{\prime}}(y ; M)$ all y, since the space of strategies available increases when $\varepsilon$ decreases. Therefore, $y_{1}^{\varepsilon}$ decreases with $\varepsilon$, while $y_{2}^{\varepsilon}$ increases with $\varepsilon$. Let $y_{\mathfrak{i}}$ be the limit of $y_{i}^{\varepsilon}$ when $\varepsilon$ goes to zero.

Now let $\varepsilon_{k}, k=1, \ldots$ be a sequence of positive numbers converging to zero. Consider the sequence $x^{k}(y)$ of optimal policies associated with $h_{\varepsilon_{k}}(y ; M)$. Considering Lemma 4 , two nonexclusive possibilities may arise: either there exists an infinite subsequence (same notation) along which $z_{1}^{\varepsilon}=z_{2}^{\varepsilon}$, or there exists an infinite subsequence (same notation again) a long which $z_{1}^{\varepsilon}<z_{2}^{\varepsilon}$. In the first case, let $c$ be an accumulation point of the $c^{\varepsilon k}$, and $x(y)$ be defined as a solution, limit of $x^{\varepsilon}(y)$, of (3.14) on $\left(y_{1}, y_{2}\right)$. In the second case, simflarly take an accumulation point of $\left(c_{1}^{\varepsilon k}, c_{2}^{\varepsilon k}, z_{1}^{\varepsilon k}, z_{2}^{\varepsilon k}\right)$ and let $x(y)$ be the limit of $x^{\varepsilon}(y)$, solution of (3.14) in $\left(y_{1}, z_{1}\right]$ with constant $c_{1}$, equal to zero in $\left[z_{1}, z_{2}\right]$, and solution of $(3.14)$ in $\left(z_{2}, y_{2}\right)$, with constant $c_{2}$.

Let the upper 1 imit of $h_{\varepsilon_{k}}(y ; M)$ be denoted by: 
$H(y ; M)=E\left[\int_{0}^{\tau} \frac{e^{-\bar{\delta} t}}{a} d t+e^{-\bar{\delta} \tau} M y_{\tau}^{a}\right] ;$

where $d y=x(y) d b+r(y+\lambda-1) d t$

and $\tau=$ first time $y_{t}$ leaves the interval $\left(y_{1}, y_{2}\right)$.

By Lemma $4, H(y: M)=h(y ; M)$.

The next step is to prove that $h(y ; M)$ is strictly increasing in $y$, on $\left(y_{1}, y_{2}\right)$. Recall that:

$$
\begin{aligned}
& h(y ; M)=\sup _{\tau,\left(x_{t}\right)} E\left[\int_{0}^{\tau} \frac{e^{-\delta t}}{a} d t+e^{-\bar{\delta} \tau} M y_{\tau}^{a}\right] \\
& d y_{t}=x_{t} d b+r\left(y_{t}+\lambda-1\right) d t
\end{aligned}
$$

where the supremum is taken over all the non-anticipatory strategies $\tau$, $\left(x_{t}\right)$. Let $y$ be in $\left(y_{1}, y_{2}\right), y \neq 1-\lambda$, and for any random event $w$, let $\left.\left(x_{t}^{y}(w)\right), \tau^{y}(w)\right)$ be the optimal strategy followed by the consumer. Let $y^{\prime}>y$, and for each $w$, consider the value obtained by the agent starting at $y^{\prime}$ when he uses the non-anticipatory strategy $\left(x_{t}^{y}(w)\right), \tau^{y}(w)$. When $\tau^{y}(w)=+\infty$, he gets $1 / a \bar{\delta}$, with both initial conditions. When $\tau^{y}(w)<+\infty$, a case that occurs with positive probability since $y \neq 1-\lambda$, the agent gets

$$
\int_{0}^{\tau} \frac{\tau^{y}(w)}{a} d t+e^{-\bar{\delta} t} \tau^{y}(w) M y_{\tau}^{\prime} \tau^{y}(w)
$$

where $y_{\tau}^{\prime} y(w)$ is the value taken by the solution of the deterministic differential equation

$$
d z=x_{t}^{y}(w) d b(w)+r(z+\lambda-1) d t
$$

at date $t=\tau^{y}(w)$; it is strictly bigger than $y_{\tau} y(w)$ since at date 0 , 
$y^{\prime}>y$ and at each date $t d z-d z^{\prime}=r\left(z-z^{\prime}\right) d t$. Since $H(y ; M)=M y^{a}$ on the complement of $\left(y_{1}, y_{2}\right)$ in $\left[y^{\ell}, y^{h}\right]$, this proves that $h(y ; M)$ is strictly increasing on $\left[y^{\ell}, y^{h}\right]$.

To complete the proof, we show that the domain $\left[z_{1}, z_{2}\right]$ on which $x(y)$ is equal to zero is at most reduced to a point. In fact, if not, $h(y ; M)$ would be twice continuously differentiable on $\left(z_{1}, z_{2}\right)$ and by Ito's formula, one would have

$$
\sup _{x \geq 0}\left[\frac{\sigma^{2}}{2} h^{\prime \prime} x^{2}+(r y+x \mu-r(1-\lambda)) h^{\prime}-\bar{\delta} h+\frac{1}{a}\right]=0
$$

and the sup would be obtained for $x=0$. This implies $h^{\prime \prime} \leq 0, h^{\prime} \leq 0$, a contradiction with the fact that $h$ is strictly increasing.

Finally, since $x(y)>0$ for a $11 y$ in $\left[y_{1}, y_{2}\right]$ except at $z=z_{1}=z_{2}, h(y ; M)$ is twice continuously differentiable on $\left(y_{1}, y_{2}\right)$ except at $z$. Taking 1 imits at $y_{1}$ and $y_{2}$, using the continuity of $x(y)$ and the fact, that $h(y ; M) \geq$ Mya with equality at $y_{1}, y_{2}$, gives

$$
x(y) \geq \frac{\mu y_{i}}{\sigma^{2}(1-a)} \quad i=1,2
$$

Let $y^{*}$ be the point in $\left[y_{1}, y_{2}\right]$ which maximizes $h(y ; M)-M y^{a}$. We have:

$$
h^{\prime}\left(y^{*} ; M\right)=a M y * a-1
$$

and

$$
\lim _{y \rightarrow y^{\star}} h^{\prime \prime}(y ; M) \leq a(a-1) M y^{\star^{a-2}}
$$

This implies

$$
x\left(y^{*}\right) \leq \frac{\mu y^{*}}{\sigma^{2}(1-a)} .
$$

Therefore the function $x(y)$ is above the half line of equation $x=\mu y /$ $\sigma^{2}(1-a)$ for $y=y_{1}$ and $y=y_{2}$, and under this half line at $y=y^{*}$ in 
$\left(y_{1}, y_{2}\right)$. This implies that $x(y)$ is of the type described in Lemma $3(i i)$, which in turn shows that $z$ must be equal to $(1-\lambda)$. Q.E.D.

This completes the first part of the proof. We enter the second part of the proof which consists in looking for a value of $M$ that satisfies (2.12) and in getting rid of the inequality $y^{h} \geqslant y \geqslant y^{\ell}$.

The following property is going to be usefur.

Lemma 6: Consider the stochastic differential equation

$$
d y=x(y) d b+r(y+\lambda-1) d t
$$

where $x(y)$ is a solution of (3.11) satisfying Lemma 2(ii), for some initial condition $y_{0}$, in $\left(y_{1}, y_{2}\right)$.

If $y_{0}<1-\lambda$, then $y$ reaches $y_{1}$ in finite time with a strictiy positive probability.

If $y_{0}>1-\lambda$, then $y$ reaches $y_{2}$ in finite time with a strictly positive probability.

If $y_{0}=1-\lambda$ and $x\left(y_{0}\right)>0$, then $y$ reaches either $y_{1}$ or $y_{2}$ in finite time with a strictly positive probability.

Proof of Lemma 6: It follows from a standard property of regular diffusion processes: If $y_{0}<1-\lambda$, there is an open interval containing yo, say $\left(y_{1}, \bar{y}_{1}\right)$, on which $x(y)>\varepsilon>0$. Therefore, the probability that $y$ hits the boundaries of this interval in finite time is equal to one, and the probability that $y$ hits $y_{1}$ before $\bar{y}_{1}$ is strictly positive (adapt e.g. Karlin and Taylor [1981, chapter 15]). A similar argument applies for $y_{2}$ when $y_{0}>1-\lambda$. Q.E.D.

To each $M$ satisfying (2.13), one can associate by Lemma 5 an interval $\left(y_{1}(M), y_{2}(M)\right)$ in which the solution $h(y ; M)$ of (2.9) under (2.10) and (2.11) is such that $h(y ; M)>M y^{a}$. Note that $y_{1}(M), y_{2}(M), h(y ; M)$ are 
implicitly functions of $\lambda$. We write $h_{\lambda}(y ; M)$ to make this dependence explicit. To go back to the original problem, an intermediate step is to study how $h_{\lambda}(y ; M) / M$ vartes with $\lambda$, holding $M$ fixed.

Lemma 7: For $M<(1-\lambda)^{-a} v$, or $a>0$ (resp. $a<0$ ), if $y$ is in $\left(y_{1}(M), y_{2}(M)\right)$, except $y \neq 1-\lambda$ if $x(1-\lambda)=0$, then $h_{\lambda}(y ; M) / M$ is strictly decreasing (resp. increasing) in $M$ and strictly increasing (resp. decreasing) in $\lambda$. Furthermore, if $y$ is in $\left\{y(M)+\lambda, 2^{y}(M)+\lambda\right)$ except $y \neq 1-\lambda$ if $x(1-\lambda)=0$, then $h_{\lambda}(y-\lambda ; M) / M$ is strictly decreasing (resp. increasing) in $\lambda$.

Proof of Lemma 7: We give the proof when $a$ and $M$ are positive. The case $a<0, M<0$ can be handled along the same lines. When $M>0$, we have:

$$
\begin{aligned}
\frac{1}{M} h_{\lambda}(y ; M) & =\sup _{\tau_{1}\left(x_{t}\right)} E\left[\int_{0}^{\tau} \frac{e^{-\bar{\delta} t}}{a M} d t+e^{-\bar{\delta} \tau} y_{\tau}^{a}\right] \\
d y_{t} & =x_{t} d b+r\left(y_{t}+\lambda-1\right) d t
\end{aligned}
$$

where the supremum is taken over all the nonanticipatory strategies $\tau,\left(x_{t}\right)$. We use an argument similar to the proof that $h_{\lambda}(y ; M)$ is increasing in $M$, in Lemma 5.

Given $y$ in $\left(y_{1}(M), y_{2}(M)\right)$, and any random event $w$, let $\left(x_{t}^{y}(w)\right.$, $\left.\tau^{y}(w)\right)$ be the optimal nonanticipatory strategy followed by the consumer. $\tau^{y}(w)$ is strictly positive with probability 1 . Therefore, if $M$ is decreased to $M^{\prime}$, applying the same strategy leads to a higher value than $h_{\lambda}(y ; M) / M$, since the first term in the expectation is strictly increasing and the second term is unchanged. Therefore:

$$
h_{\lambda}\left(y ; M^{\prime}\right) / M^{\prime}>h_{\lambda}(y ; M) / M \text {. }
$$


Similarly, consider $\lambda^{\prime}>\lambda$. For all $y, y \neq 1-\lambda$ if $x(1-\lambda)=0$, by Lemma $6, \tau^{y}(w)$ is finite with positive probability. Consider an event $w$ such that $\tau^{\mathrm{y}}(w)<+\infty$. Then applying the $\lambda$ optimal strategy when $\lambda^{\prime}$ prevails gives a higher value to $y_{\tau}$, and therefore the desired result. This shows that $h_{\lambda}(y, M) / M$ is strictly increasing for all $y, y \neq 1-\lambda$ if $x(1-\lambda)=0$.

Finally, we study the function $h_{\lambda}(y-\lambda ; M)$. By definition:

$$
\begin{gathered}
\frac{1}{M} h_{\lambda}(y-\lambda ; M)=\sup _{\tau,\left(x_{t}\right)} E\left[\int_{0}^{\tau} \frac{e^{-\bar{\delta} t}}{a M} d t+e^{-\bar{\delta} \tau} y_{\tau}^{a}\right] \\
d y_{t}=x_{t} d b+r\left(y_{t}+\lambda-1\right) d t \\
y_{0}=y-\lambda .
\end{gathered}
$$

When $\lambda$ decreases, the initial condition increases, but $\left(y_{0}+\lambda\right)$ stays constant and, since the differential equation can be rewritten

$$
\begin{aligned}
& d\left(y_{t}+\lambda\right)=x_{t} d b+r\left(y_{t}+\lambda-1\right) d t \\
& \text { for any event } w, y_{t}(w, \lambda)+\lambda \text { is equal to } y_{t}\left(w, \lambda^{\prime}\right)+\lambda^{\prime} .
\end{aligned}
$$

Therefore, for all $y$, such that $y \neq 1-\lambda$ if $x(1-\lambda)=0$, we have for the $\lambda$ optimal strategy starting at $y$ :

$$
\lambda^{\prime}<\lambda \text { implies } y_{\tau}\left(w, \lambda^{\prime}\right)=y_{\tau}(w, \lambda)+\lambda-\lambda^{\prime}>y_{\tau}(w+\lambda) .
$$

Consequently

$$
\frac{1}{M} h_{\lambda^{\prime}}\left(y-\lambda^{\prime} ; M\right)>\frac{1}{M} h_{\lambda}(y-\lambda ; M) \text {, since } \tau^{y}(w) \text { is finite with strictly }
$$

positive probability by Lemma 6 .

To go back to the original problem and characterize the function $h(y)$, we have to determine how $M$ varies with $\lambda$ through equation (2.12), which can be rewritten 
(A.13) $\quad 1=\sup _{z} z^{-a} h_{\lambda}(z-\lambda ; M) / M$

when $a$ is positive, the sup being taken on $\left\{z \mid y^{\ell}+\lambda \geqslant z \geq y^{h}+\lambda\right\}$. Note that the sup is replaced by an inf when $a<0$.

Lemma 8: The function $h(y)$ which solves (3.1) to (3.4) is continuous, strictly increasing in $y$, continuously differentlable except perhaps at $y=1-\lambda$. There exists an interval $\left(y_{1}^{\lambda}, y_{2}^{\lambda}\right)$ and a number $M_{\lambda}$ such that:

(i) $h(y)>M_{\lambda} y^{a}$ on $\left(y_{1}, y_{2}\right)$ and $h(y)$ is twice continuously differentiable on $\left(y_{1}, y_{2}\right)$ except perhaps at $y=1-\lambda$. $\bar{y}=(1-\lambda)(1-a) r / B$ belongs to $\left(y_{1}, y_{2}\right) . M_{\lambda}$ is strictly decreasing in $\lambda$, when it is larger than $1 / \bar{\delta} a, y_{1}^{\lambda}$ is strictly decreasing in $\lambda$, and $y_{2}^{\lambda}$ is strictly increasing in $\lambda$.

(ii) $h(y)=M_{\lambda} y^{a}$ in the complement in $R^{+}$of $\left(y_{1}, y_{2}\right)$

\section{Proof of Lemma 8: Let}

$$
H(\lambda, M)=\sup _{z} z^{-a} n_{\lambda}(z-\lambda ; M) / M
$$

When $a>0$, from $(A .12), H(\lambda, M)$ tends to to when $M$ tends to zero. It is equal to $\left(y^{h} /\left(y^{h}+\lambda\right)\right)^{a}$ for $M>(1-\lambda)^{-a} v$. It is continuous in $M$ by Lemma 1 and by the theorem of the maximum. Furthermore, by Lemma 7, if the maximizer $z^{*}$ is different from 1 or $x(1-\lambda)>0, H$ is strictly decreasing in $M$ and in $\lambda$. Therefore, for $\lambda$ fixed, the solution in $M$ of equation (A.13)

$$
H(\lambda, M)=1
$$

is unique and a strictly decreasing function of $\lambda$. If $z^{*}=1$ and $x(1-\lambda)=0, H(\lambda, M)=1 / a \bar{\delta} M$ and therefore $M=1 / \bar{\delta} a$. By construction, aga in for a $>0$ : 


$$
h_{\lambda}\left(y, M_{\lambda}\right) / M_{\lambda}>y^{a} \quad \text { on } \quad\left(y_{1}, y_{2}\right)
$$

By Lemma 7 , the left-hand side is strictly increasing in $\lambda$ (except perhaps at $y=1-\lambda)$, which proves that the interval $\left(y_{1}, y_{2}\right)$ increases with $\lambda$.

To conclude the proof, we have to show that the solutions that we have found satisfy (A.4), i.e.:

Lemma 9: For a11 $\lambda, 0 \leqslant \lambda<1$, there exists $y^{h}>y^{l}>0$ such that:

$$
\begin{aligned}
& y_{1}^{\lambda}>y^{\ell} \\
& y_{2}^{\lambda}<y^{h}
\end{aligned}
$$

Proof of Lemma 9: We use the fact that for all $y \neq 1-\lambda, h_{\lambda}$ is twice continuously differentiable and

$$
\frac{h^{\prime \prime}(y)}{h^{\prime}(y)}=\frac{-\mu}{\sigma^{2} x(y)} \quad y \neq 1-\lambda \quad y_{1} \leqslant y \leqslant y_{2}
$$

Furthermore:

$$
x(y) \geqslant \operatorname{Max}\left(\theta_{1}(y+\lambda-1), \theta_{2}(y+\lambda-1)\right)
$$

where $\theta_{1}<0$ and $\theta_{2}>0$ are the two roots obtained from (3.13). Straightforward integrations, with starting points $y_{1}$ and $y_{2}$, and $h^{\prime}\left(y_{j}\right) / h\left(y_{j}\right)=a / y_{j}$ lead to:

for $1-\lambda<y \leqslant y_{2}$

$$
h(y) \geqslant h\left(y_{2}\right)\left[1+\frac{a}{y_{2} \tau_{2}}(y+\lambda-1)^{1-\tau 2}\left[(y+\lambda-1)^{\tau} 2-\left(y_{2}+\lambda-1\right)^{\tau 2}\right]\right]
$$

where $\tau_{2}=1-\frac{\mu}{\sigma^{2} \theta_{2}}$ is positive (from the second degree equation

defining $\theta_{2}$ ). Similarly 
for $y_{1} \leqslant y<1-\lambda$

$$
h(y) \geqslant h\left(y_{1}\right) \cdot\left[1+\frac{a}{y_{1} \tau_{1}}\left(1-\lambda-y_{1}\right)^{1-\tau_{1}}\left[\left(1-\lambda-y_{1}\right)^{\tau_{1}}-(1-\lambda-y)^{\tau_{1}}\right]\right]
$$

where $\tau_{1}=1-\frac{\mu}{\sigma^{2} \theta_{1}}$ is also positive while $1-\tau_{1}$ is negative. Note that when we let $y$ tend to $(1-\lambda)$ in the two above inequalities we get:

$$
h(1-\lambda) \geqslant h\left(y_{i}\right)\left[1+\frac{a}{\tau_{i} y_{i}}\left(1-\lambda-y_{j}\right)\right]
$$

We know by adapting the argument of Theorem 2.1 to the case where $y$ satisfies (A.4) that

$$
h(1-\lambda) \leqslant v(1-\lambda)^{a} \text {, }
$$

and

$$
h\left(y_{1}\right) \geqslant \frac{v_{2} y_{i}^{a}}{a}
$$

To complete the proof, we examine separately $y_{1}$ and $y_{2}$.

First $y_{2}$. If $y_{2} \leqslant 1-\lambda$, one can take $y^{h}=1-\lambda$. Otherwise, (A.14) gives an upper bound for $y^{h}$. In fact, the right-hand side of the inequality tends to to when $y_{1}$ goes to $+\infty$ for $a>0$ (check that a / $\tau_{2}<1$ by definition of $\tau_{2}$, another property of the second degree equation defining $\theta_{2}$ ), and tends to zero for $a<0$.

Finaliy, $y_{1}$. Similarly, when $y_{1}$ goes to zero, $a>0$, the right-hand side of (A.14) goes to to. When $a<0, h\left(y_{i}\right) \leqslant \nu y^{a}$ by (2.13), and this implies that $h\left(y_{1}\right)$ tends to $-\infty$ when $y_{1}$ tends to zero, so that the right-hand side of (A.14) tends aiso to $+\infty$. Q.E.D.

Theorem 2.2 is then a direct consequence of Lemmas 3 and 9 . Lemmas 8 and 9 imply Theorems 3.3 and 3.4. Theorems 3.5 and 3.6 follow from Lemma 5 . 


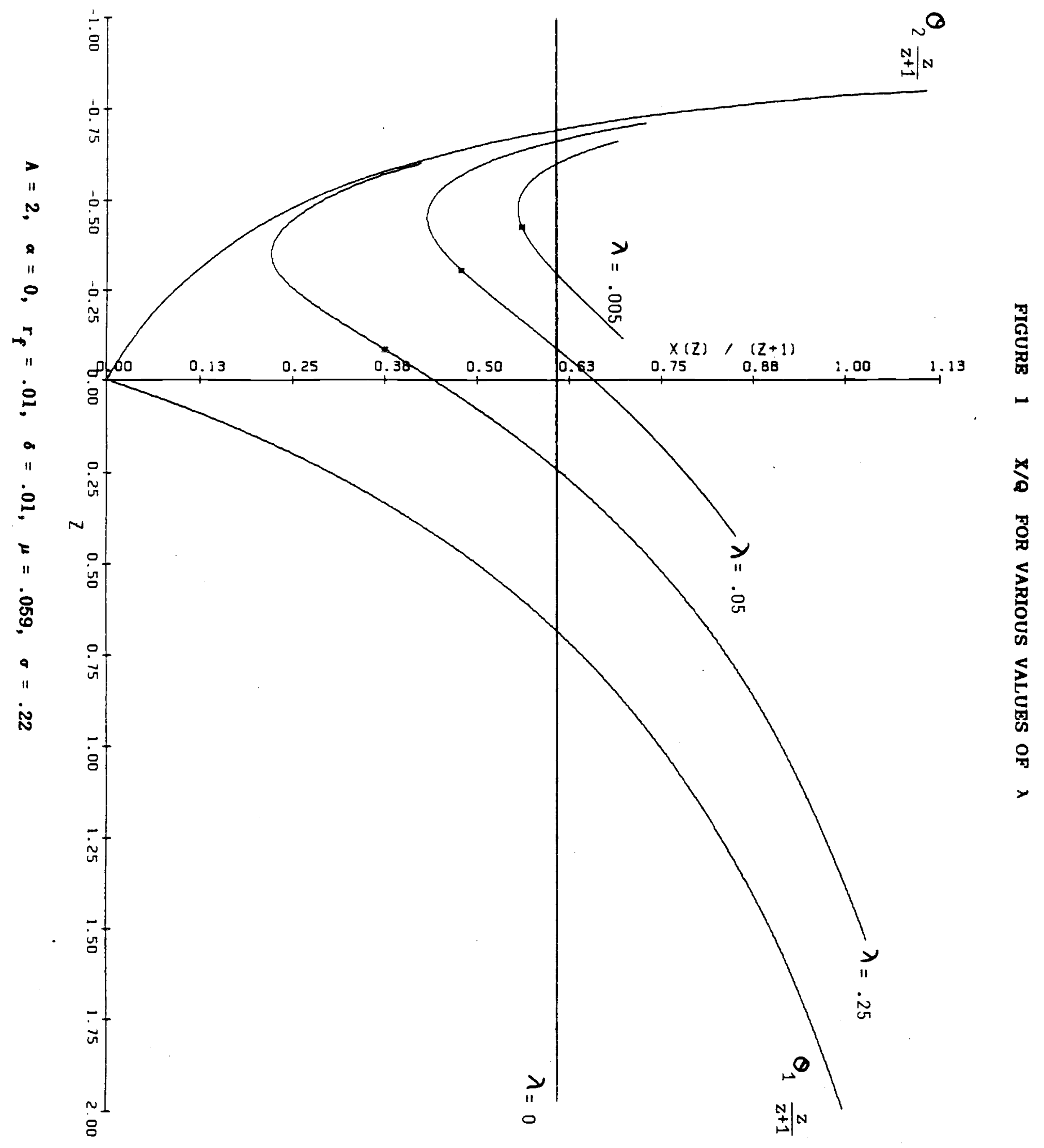




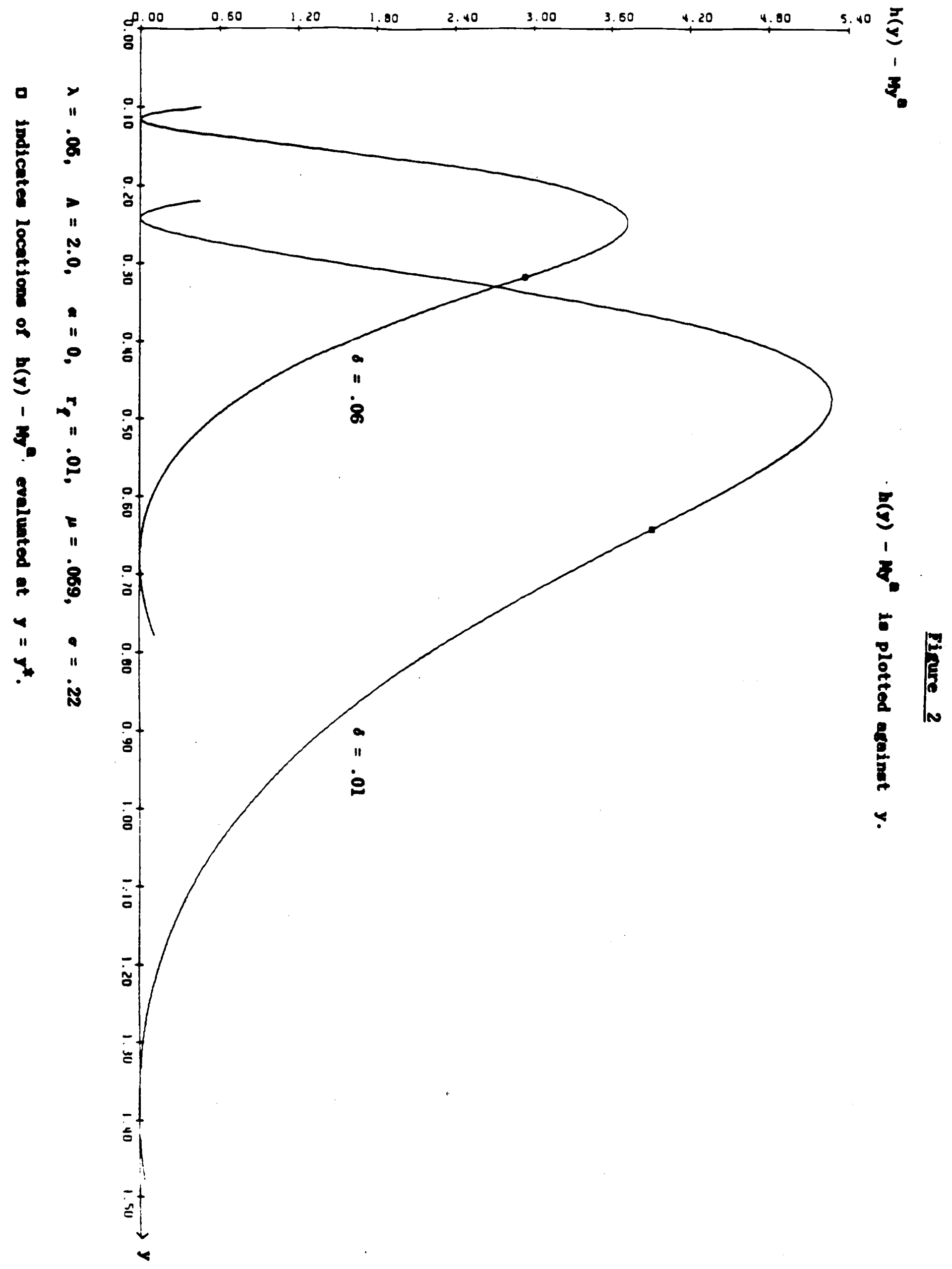

